\title{
Review zârticle
}

\section{With or without you -_ Proteomics with or without major plasma/serum proteins}

Elisabetta Gianazza1 $1^{\mathrm{a}}$ *

elisabetta.gianazza@unimi.it

Ingrid Miller $2^{\mathrm{b}}$

Luca Palazzolo1 $1^{\mathrm{a}}$

Chiara Parravicini1 $1^{a}$

Ivano Eberini $1^{\text {a }}$

aㅡㅁipartimento di Scienze Farmacologiche e Biomolecolari, Università degli Studi di Milano, Via Balzaretti 9, I-20133 MilaneMilan, Italy

끈 Institut für Medizinische Biochemie, Veterinärmedizinische Universität Wien, Veterinärplatz 1, A-1210 Wien, Austria

**Corresponding author.

\section{Abstract}

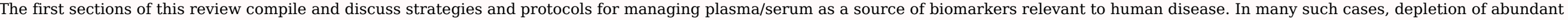

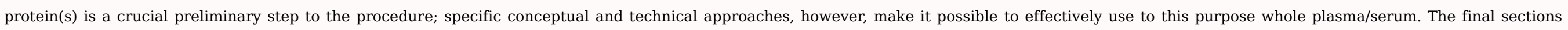

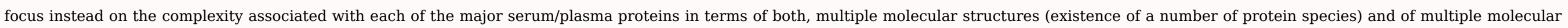

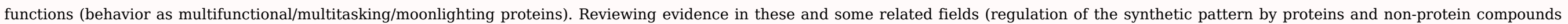

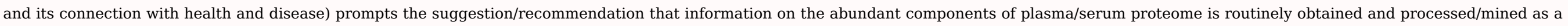
valuable contribution to the characterization of any non-physiological condition and to the understanding of its mechanisms and of its implications/sequels.

Keywords: Serum proteins; Depletion; Enrichment; Immunodepletion; Protein species

\subsection{Forewords: powers of 10}

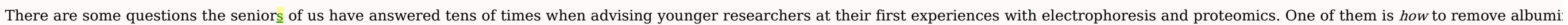

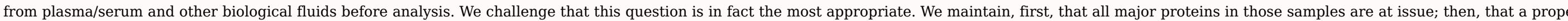

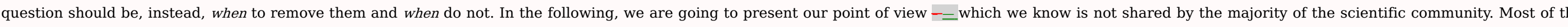
information will focus on human specimens although similar concepts and procedures apply to plasma/serum from laboratory and farm animals as well.

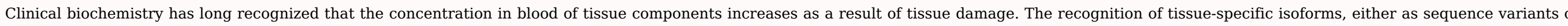

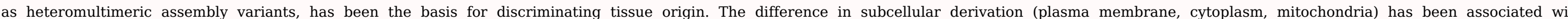

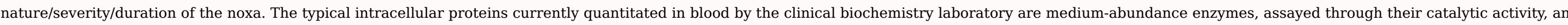
high-abundance structural proteins, identified through their immunological reactivity.

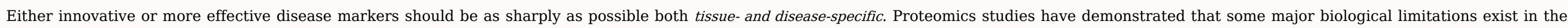

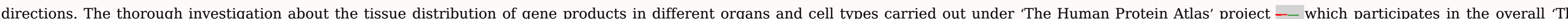




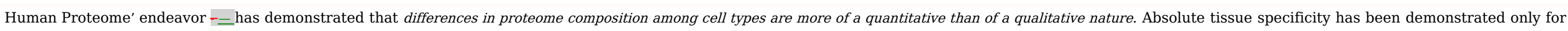

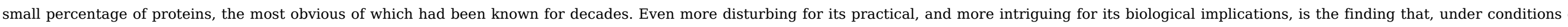
cellular stress, the changes in proteome composition hardly depend on the nature of the stress.

How does the circulating concentration of current and perspective disease markers compare with that of other proteins in plasma/serum?

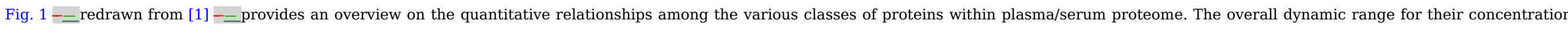

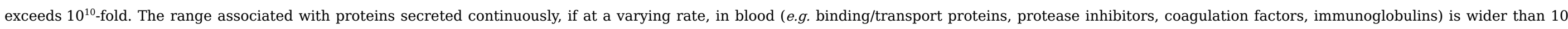

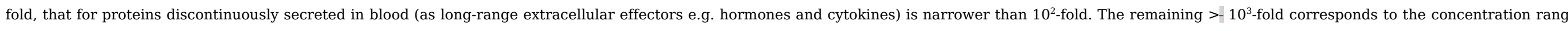
for proteins not targeted for secretion but leaking from tissues either as intact molecules or as proteolytic fragments.

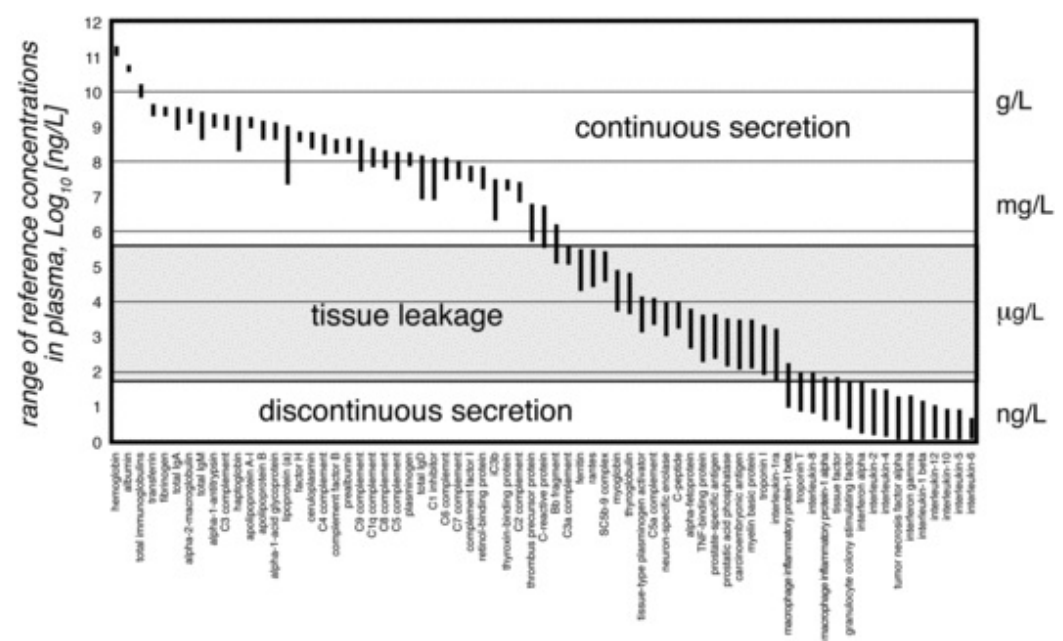

Fig. 1 A chart of the circulating concentrations of different classes of proteins found in plasma/serum. (Redrawn from [1]).

\section{alt-text: Fig. 1}

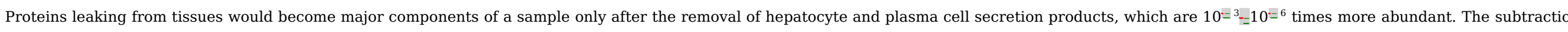

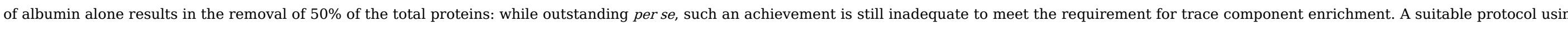

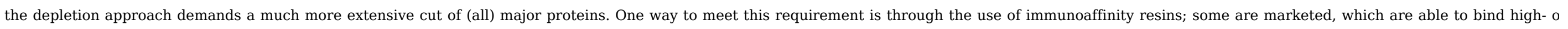
medium-abundance plasma/serum components, resulting in the depletion of up to $99 \%$ of the total proteins.

\subsection{Without major plasma/serum proteins}

\subsubsection{How to remove major plasma/serum proteins}

\subsection{Removing albumin}

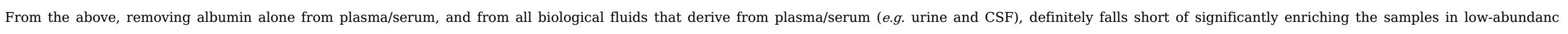

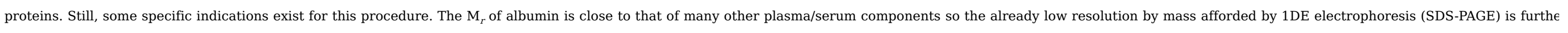

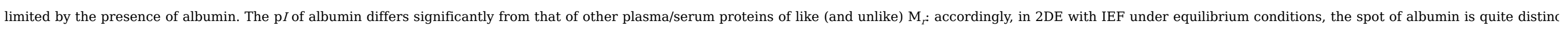

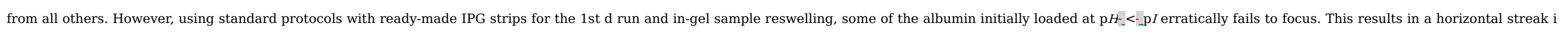
the low $\mathrm{p} H$ (anodic) region, which overlaps acidic protein spots, blurring their pattern and confusing their quantitation. Cutting albumin concentration also cuts such interference. 


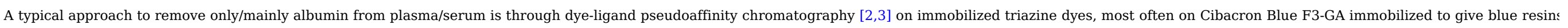

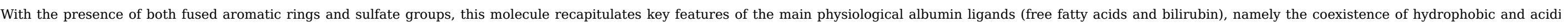

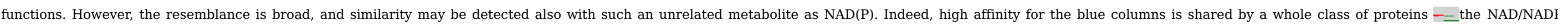

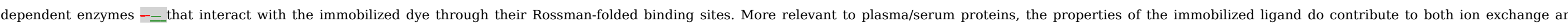

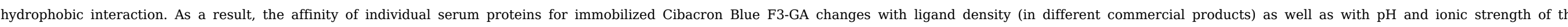

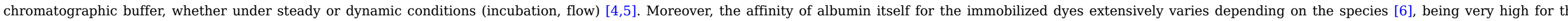

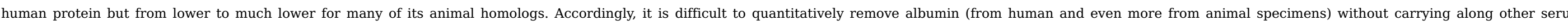

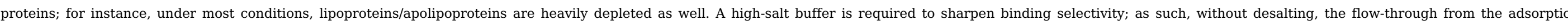

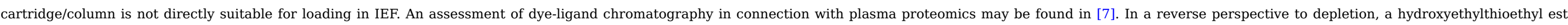

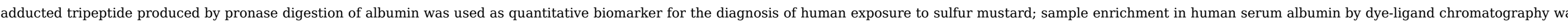
instrumental to improve the performance of the test [8].

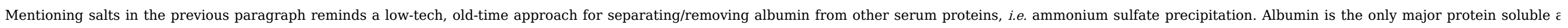

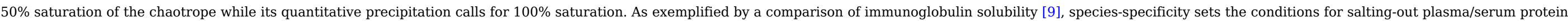

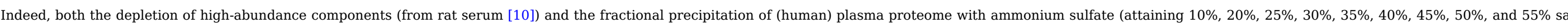
concentrations [11]) have been recently explored as preliminary steps before 2DE analysis.

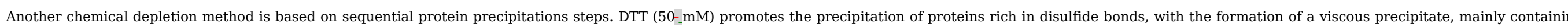

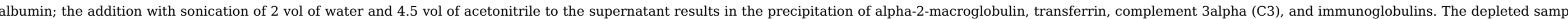
in the second supernatant may be used in proteomics experiments (Fig. 2) after evaporation of the solvent to dryness [12].

\section{treatment with dithiothreitol and acetonitrile}

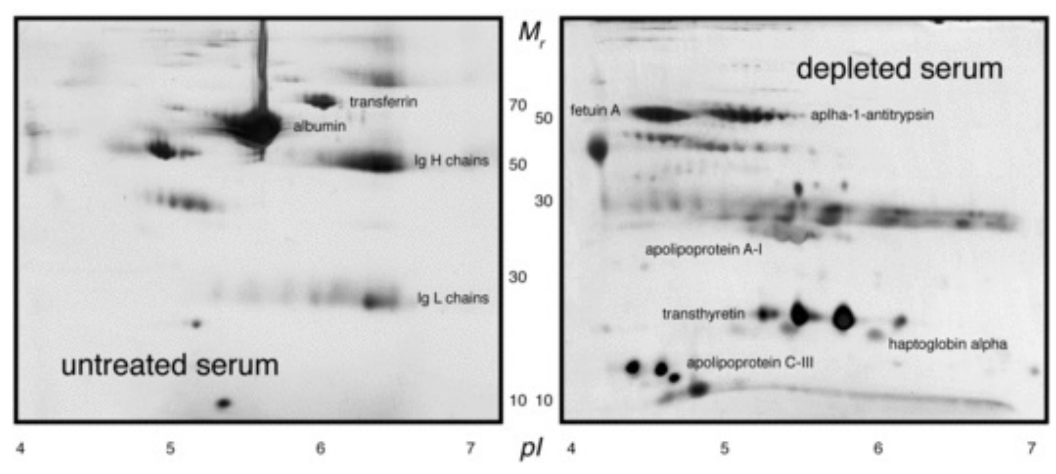

Fig. 2 2DE comparing the different protein profiles of untreated human serum and of serum depleted according to the sequential method in [12]. Protein landmarks are identified in each map. alt-text: Fig. 2

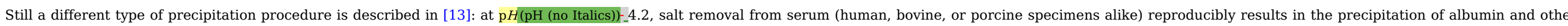
high abundance proteins (immunoglobulins, apolipoprotein A-I, transferrin, alpha-2-HS-glycoprotein, hemopexin, vitamin $D$ (vitamin $D$ (no Italics)--binding protein, serpin A3=5, complement factor B).

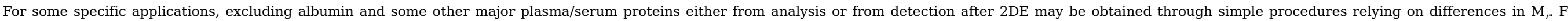

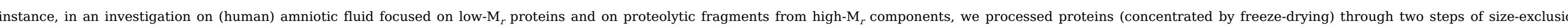

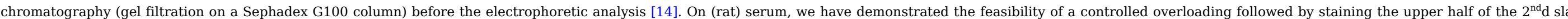




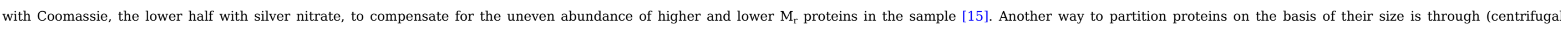

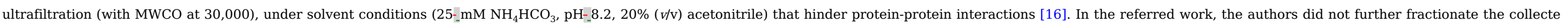
proteins but treated the whole low- $\mathrm{M}_{\mathrm{r}}$ filtrate with trypsin and analyzed the resulting peptides by SELDI.

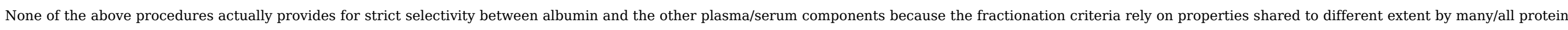

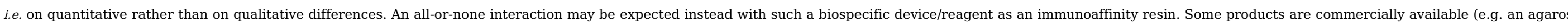

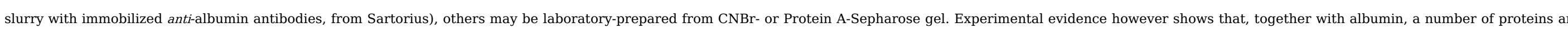

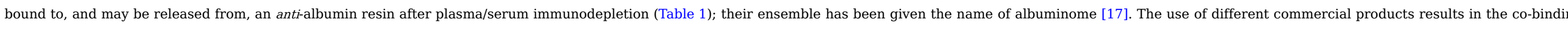

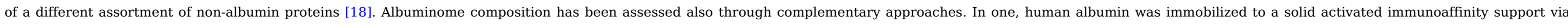

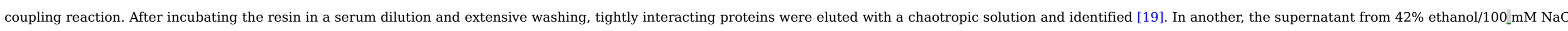

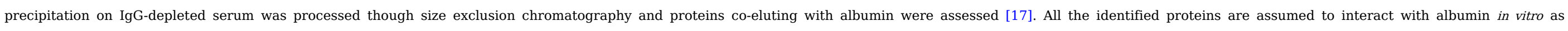

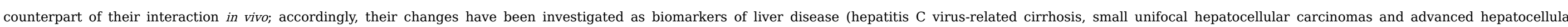
carcinomas) [20].

Table 1 Albuminome components [17].

alt-text: Table 1

Apolipoæprotein A-II

Apolipopprotein A-IV

Apolipopprotein C-II

Apolipopprotein C-III

Ceruloplasmin

Clusterin

Complement component 1 inhibitor

Intact proteins

Haptoglobin

Hemoglobin, alpha chain

Hemoglobin, beta chain

Hemopexin

Leucine-rich alpha-2-glycoprotein

Transferrin

Transthyretin

Vitamin D-binding protein

Zinc alpha-2-glycoprotein

Carboxypeptidase B2

Complement component $C 4$

Hornerin

Protein fragments

Inter-alpha-trypsin inhibitor heavy chain $\mathrm{H} 4$

Kininogen

Paraoxonase 1

Peptidoglycan recognition protein 2

Plasminogen

\subsection{Removing major plasma/serum proteins}

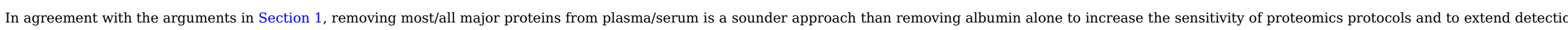

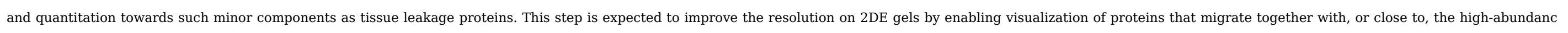
proteins and by increasing the load in the least abundant components. 


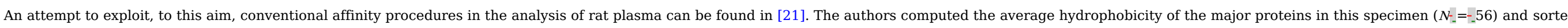

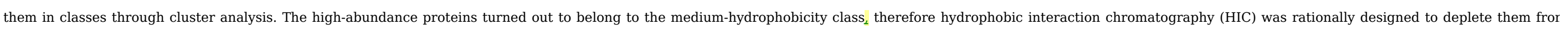
plasma. Indeed, when applied before 2DE, HIC permits to detect twice as many spots as immunoaffinity depletion of albumin (above).

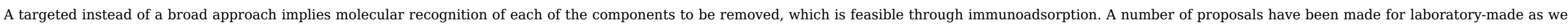

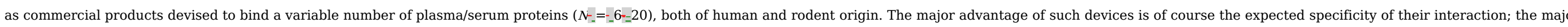

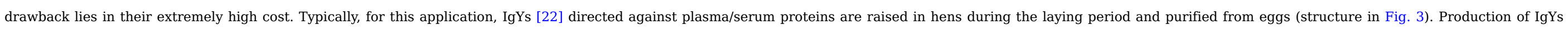

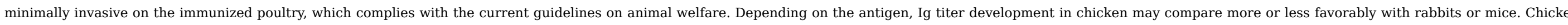

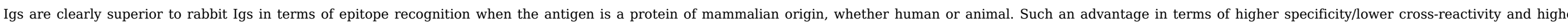

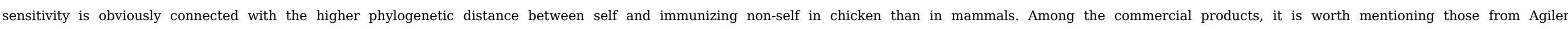

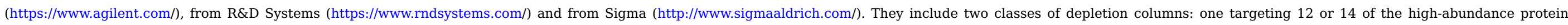

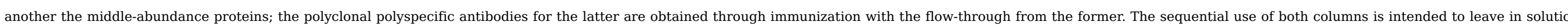
only low-abundance proteins.

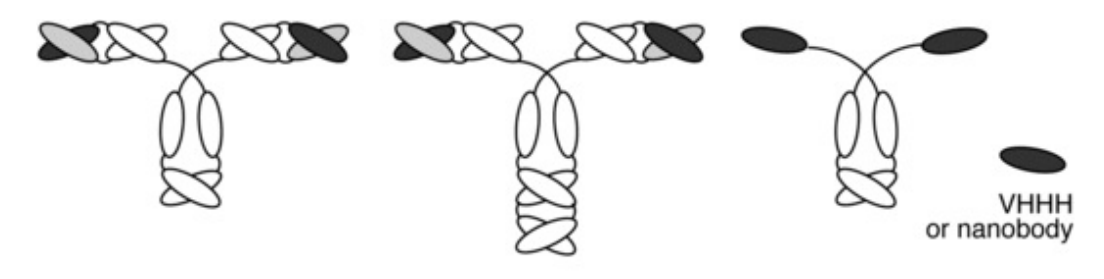

rabbit IgG

chicken $\lg Y$

camelid Ig

$\mathrm{VH} \circlearrowright \mathrm{VL} \circlearrowright \mathrm{CH} / \mathrm{L}$

Fig. 3 Overview on the structure of immunoglobulins of different animal origin.

alt-text: Fig. 3

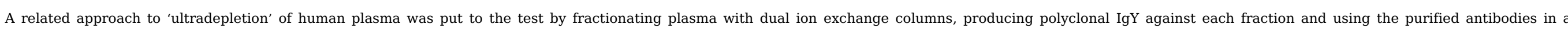
immunodepletion column. A total of 165 non-redundant proteins were identified after depletion; of these, 38 had not previously been identified in non-depleted plasma [23].

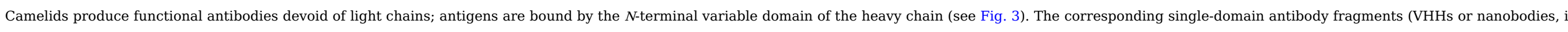

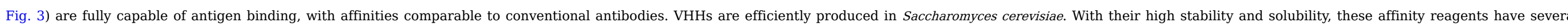

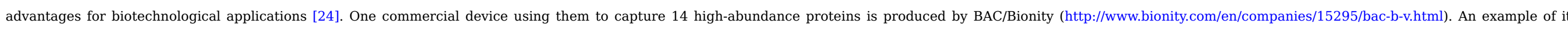
application may be found in [25] (Fig. 4): removal of high-abundance proteins is efficient and enrichment of low-abundance proteins appears reproducible from run to run. 


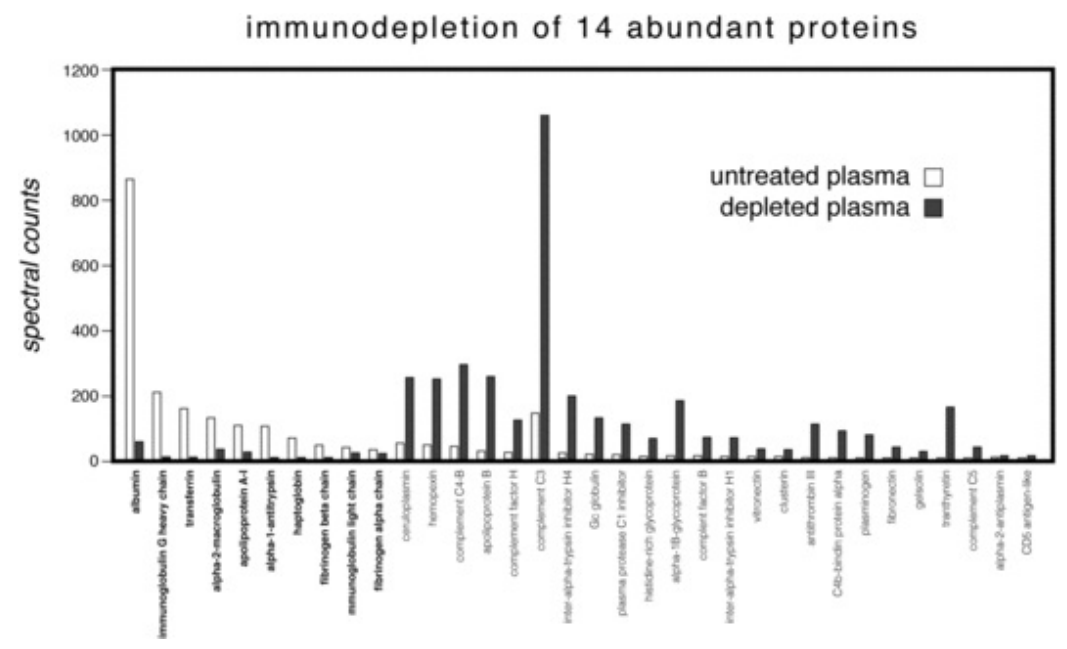

Fig. 4 Relative abundance of proteins identified in untreated plasma and in the flow-through fraction of an immunoadsorption column targeting 14 major plasma components (bold). (Redrawn from [25]). alt-text: Fig. 4

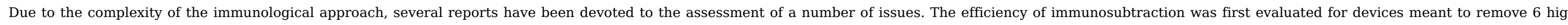

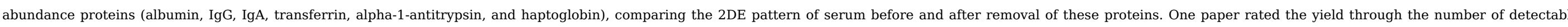

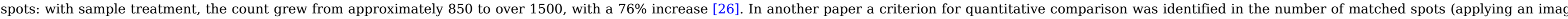
analysis software to the 2DE patterns of several replicates); the matches totaled 197 before and 317 after depletion, with an increase of 61\% [27] (Fig. 5).

immunodepletion of 6 abundant proteins

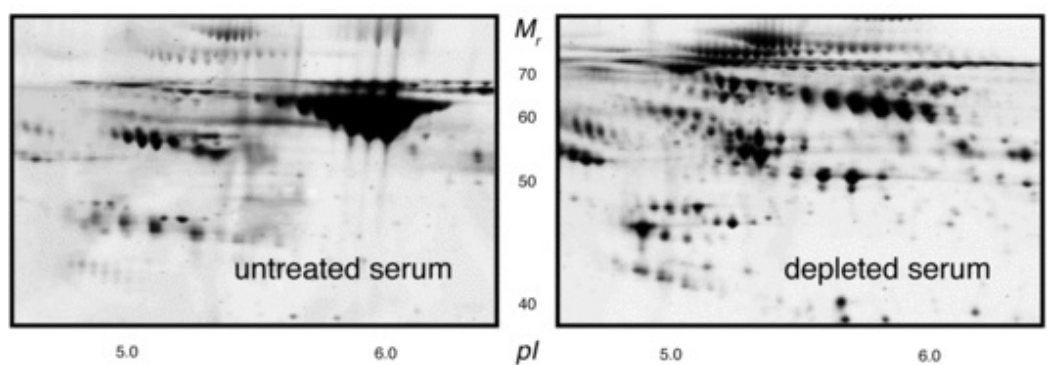

Fig. 5 2DE comparing the different protein profiles of untreated human serum and of serum immunodepleted in six high-abundance proteins (albumin, IgG, IgA, alpha-1-antitrypsin, transferrin, and haptoglobin). (From [27]). alt-text: Fig. 5

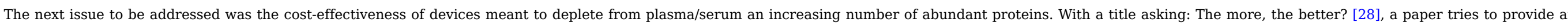

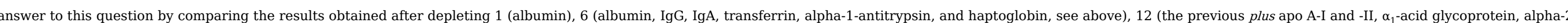

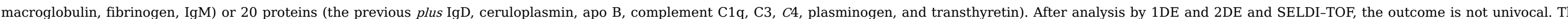

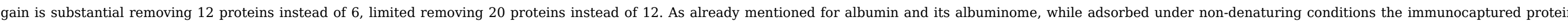

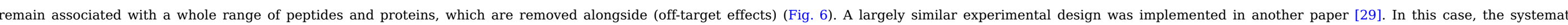

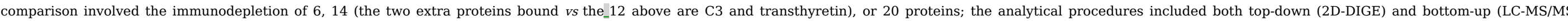

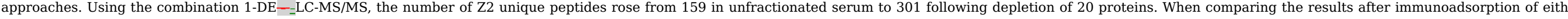




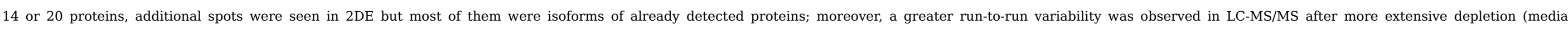

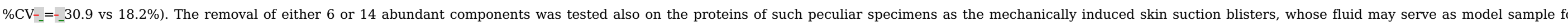

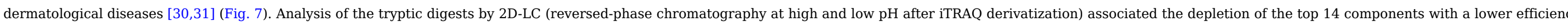

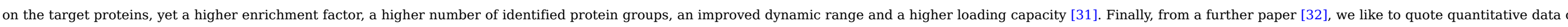

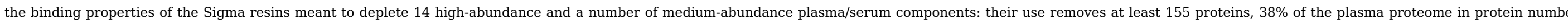

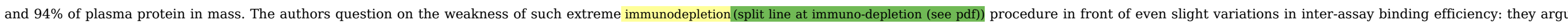
that the proteins associated with the highest percentage immunodepletion are also at the highest risk of being identified as false-positives in differential proteomic studies.

immunodepletion of 1 to 20 abundant proteins
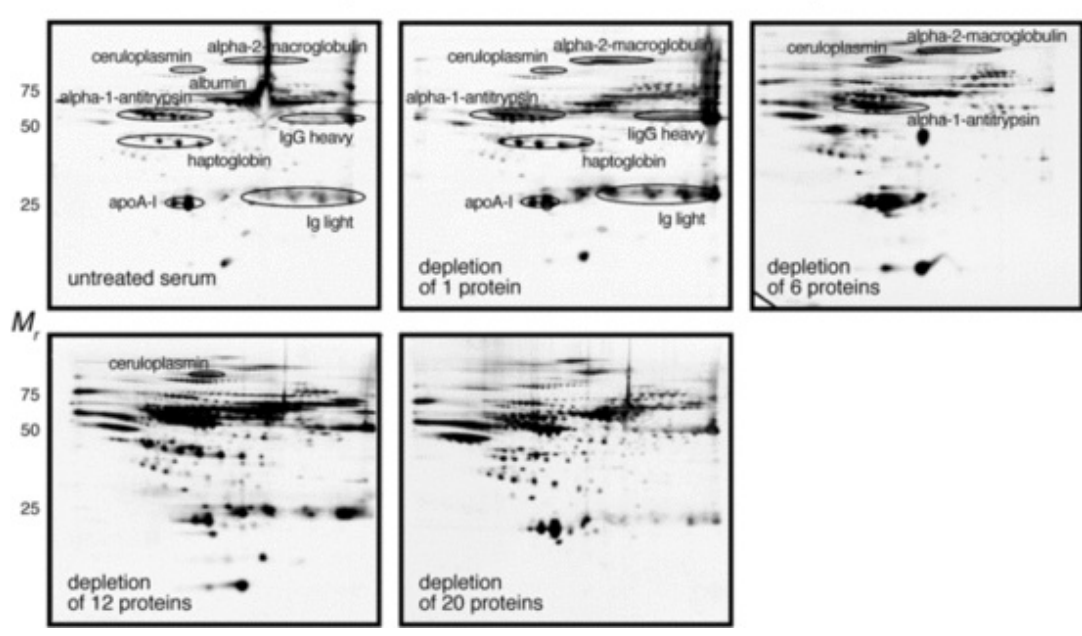

Fig. 6 2DE of untreated serum vs the unbound fractions collected after depletion of 1, 6, 12 and 20 major serum proteins using commercial columns (Table 1). A fixed amount of protein (50- $\mu$ g) was analyzed in all maps. (From [28]). alt-text: Fig. 6

\section{immunodepletion}

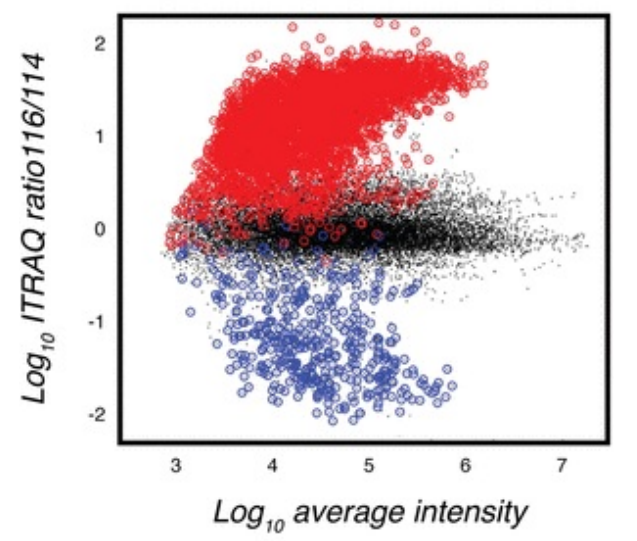

top $1-6$ plasma proteins $O$ top $7-14$ plasma proteins

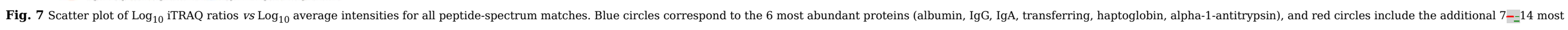


As a last line, similar to albuminome, the term depletome has been introduced to refer to the off-target immunosubtracted proteins (see above).

\subsubsection{How to enrich minor plasma/serum proteins}

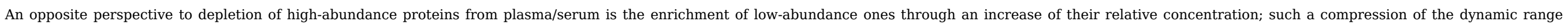

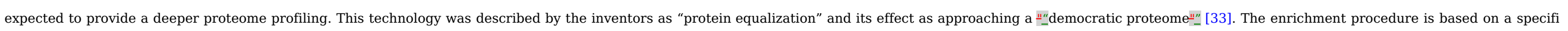

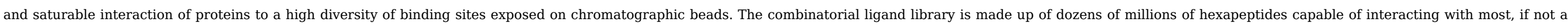

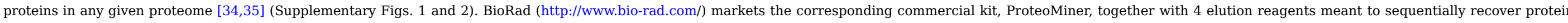

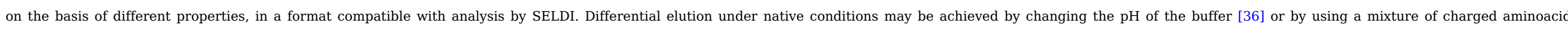

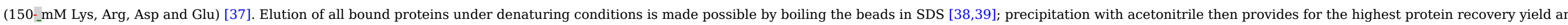
the best 2DE spot pattern [40].

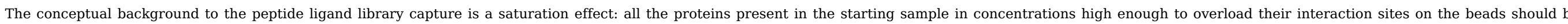

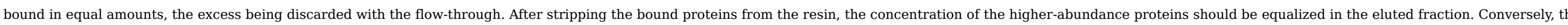

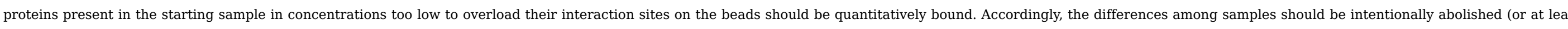

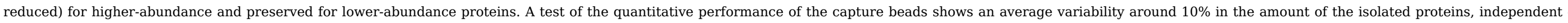

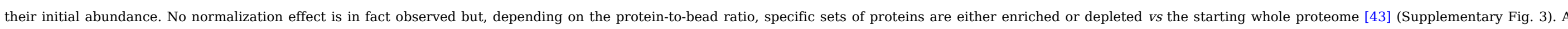

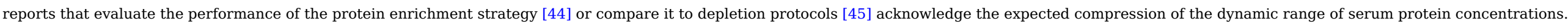

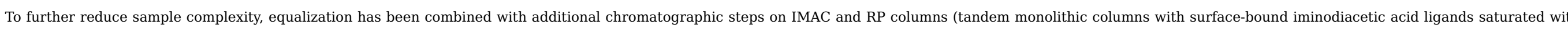

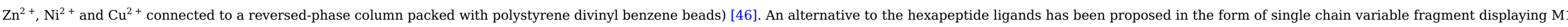

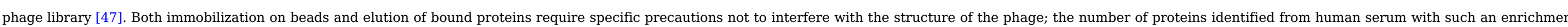
procedure is twice as high as from the untreated sample.

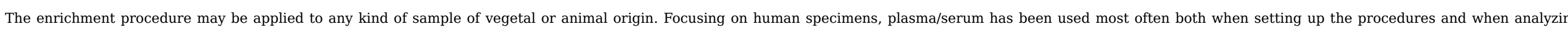

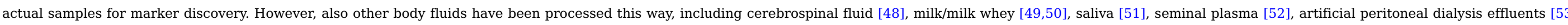
synovial fluid [54].

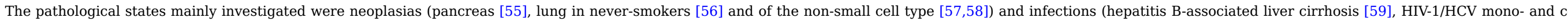

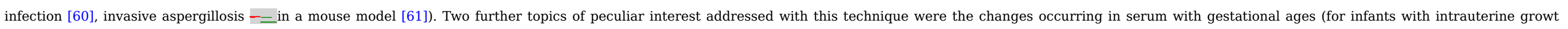
restriction [62]) and those occurring under extreme physical stress [63].

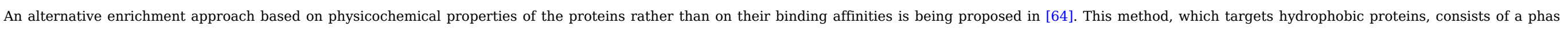

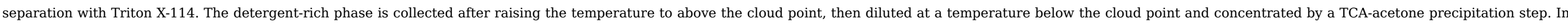

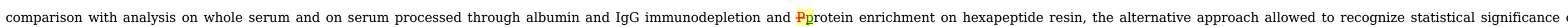

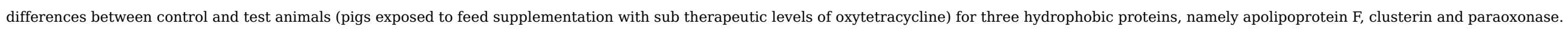

\section{3. $\underline{3}$ With major plasma/serum proteins: How to deal with whole plasma/serum}

\subsubsection{Strategies in proteomics}

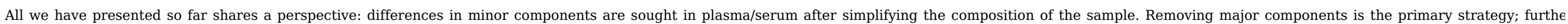




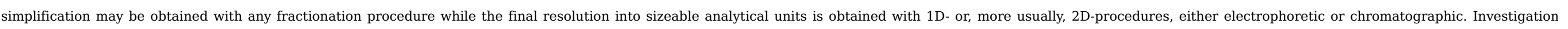

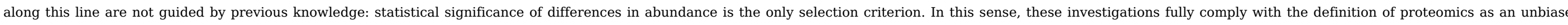
qualitative and quantitative evaluation of all protein components in a given sample - = sample in this case being human plasma/serum.

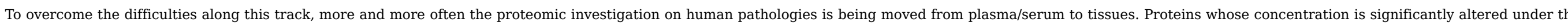

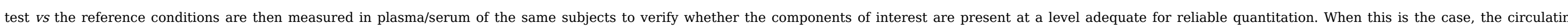
concentrations are evaluated and statistical significance of differences is finally assessed.

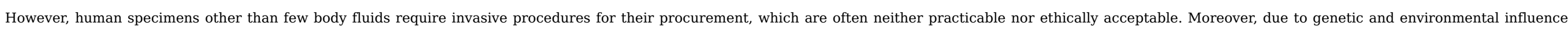

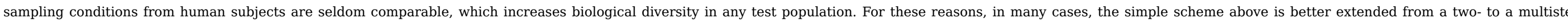

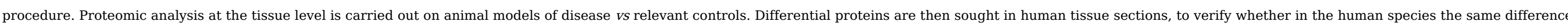
in abundance between test and control samples are present as in the animal model. Only after this check the quantitation on human plasmas/sera is eventually carried out for the putative biomarkers.

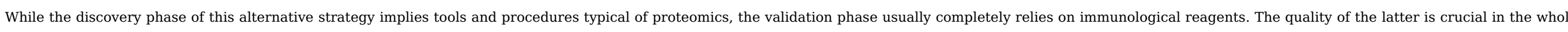
process but producing/screening high-specificity high-affinity antibodies, whether polyclonal or monoclonal, is not an easy task.

Overviews on integrated strategies for biomarker discovery and validation have been published (e.g. [65]). All of these aspects are dealt with, through relevant examples, in the following sections.

\subsection{Reference immunological reagents}

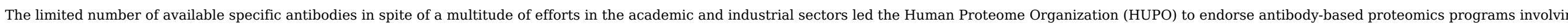
the systematic generation of antibodies for use in functional exploration of the human proteome with a high-throughput approach [66,67].

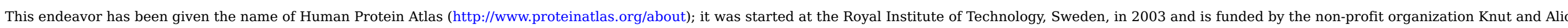
Wallenberg Foundation. Updates on the program achievements are since published on a regular basis (e.g. [68,69]).

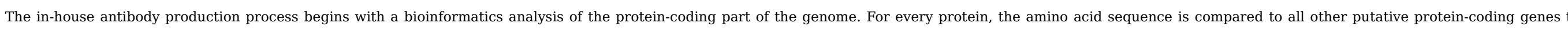

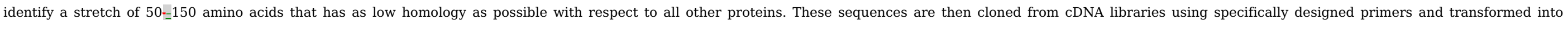

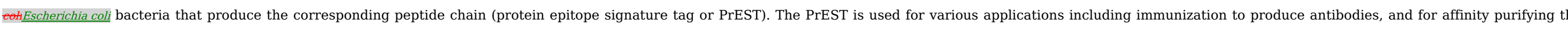

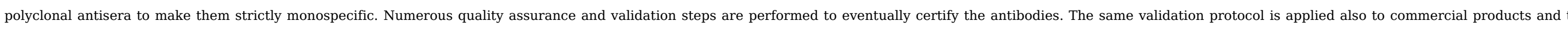
antibodies prepared by collaborating institutions.

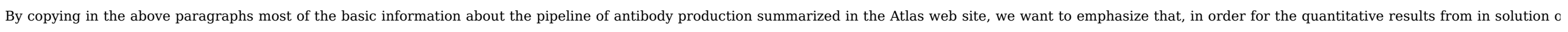

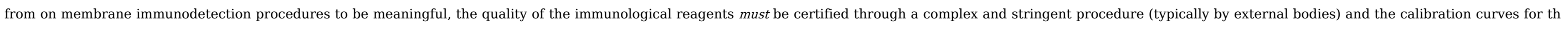

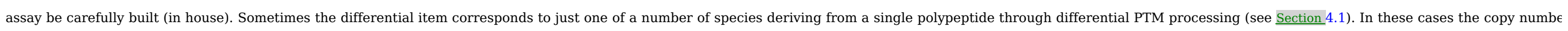

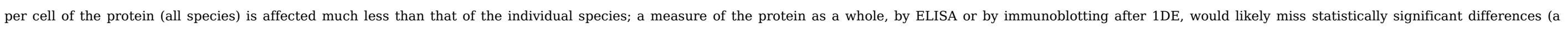
exception, 1DE after extensive proteolysis). Such cases can be effectively addressed only through 2DE with immunoblotting and comprehensive image analysis.

\subsection{Reference data on tissue proteomics}

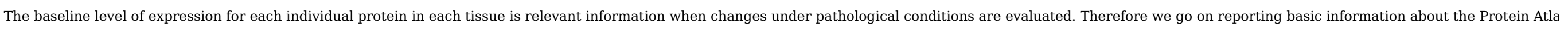
project.

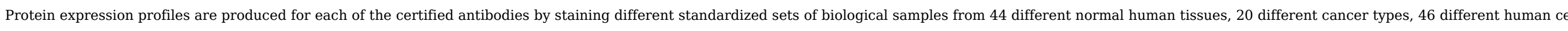

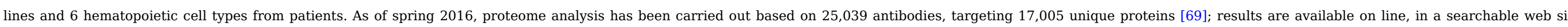

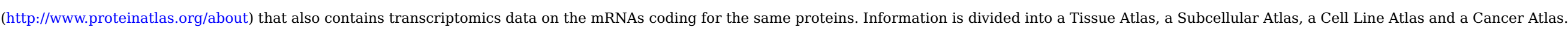




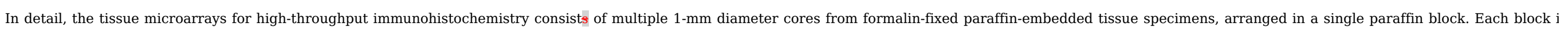

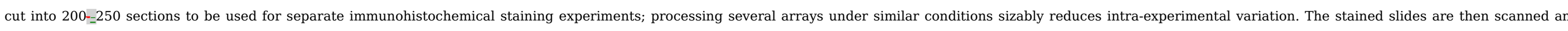
pathologists annotate each core image by scoring the intensity of immunoreactivity with its cellular localization and by counting the fraction of immunostained cells.

\subsection{Multistep experimental plans}

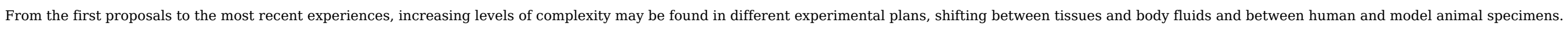

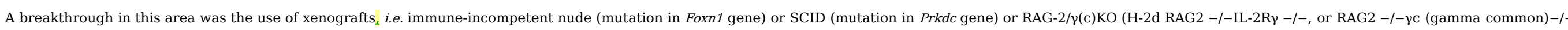

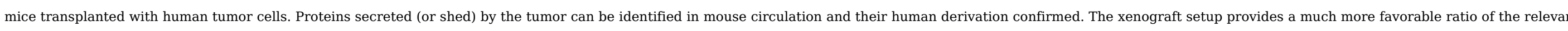

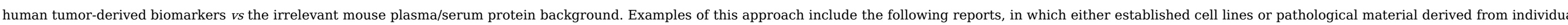
cancer patients are grafted.

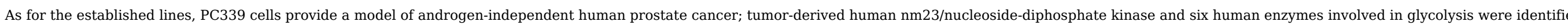

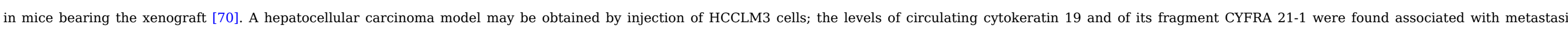
potential [71]. Three cell lines (liposarcoma [SW872], osteosarcoma [KHOS-

$$
\text { - }
$$

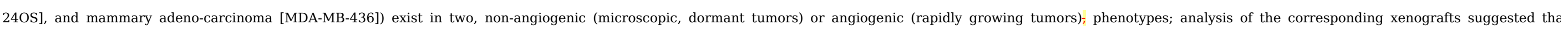

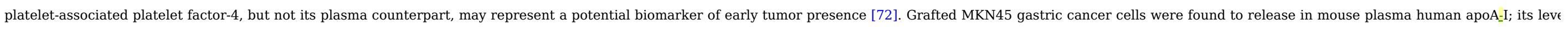
was lower in mice with large than in those with small tumors [73].

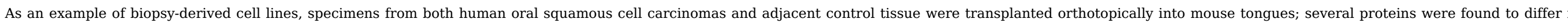
concentration between control and cancer-bearing mice; among these, EGFR levels inversely correlated with the invasive phenotype $[74,75]$.

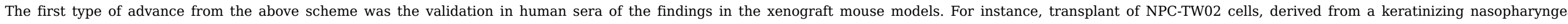

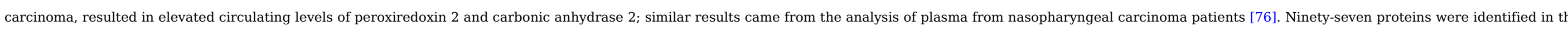

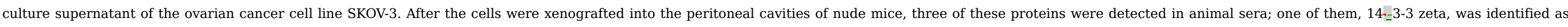

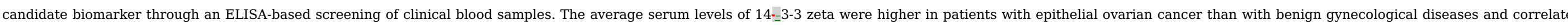

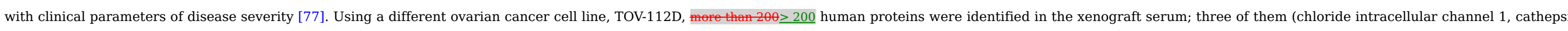
D, and peroxiredoxin 6) were then found elevated in sera from ovarian carcinoma patients [78].

A similar shift in plans applies to a different type of animal models, the genetically engineered mice.

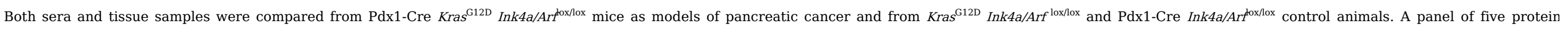

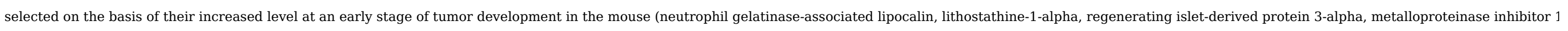

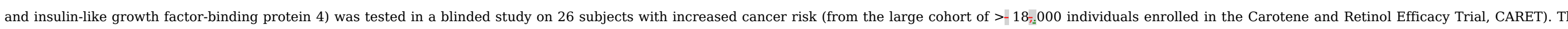

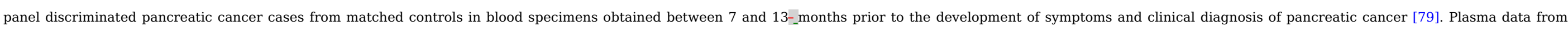

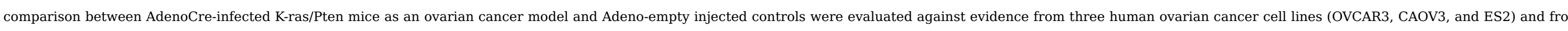

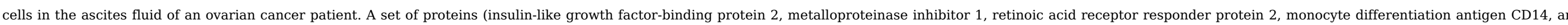
granulin) were measured at higher levels in pathological than in control tissues and body fluids, both human and murine. The difference from controls was significant from the early stages of the disease [80].

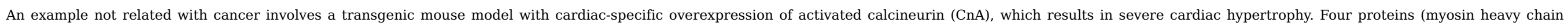

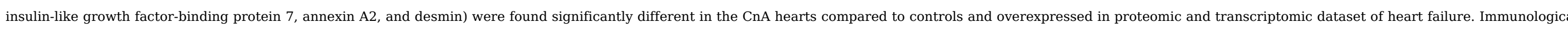
quantitation in mouse and human sera demonstrated that all four proteins increased between twofold and 150-fold in heart failure [81]. 


\subsubsection{Procedures in proteomics}

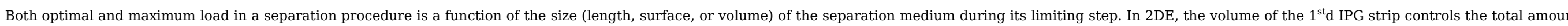

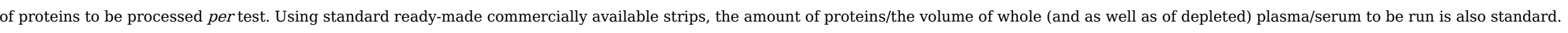

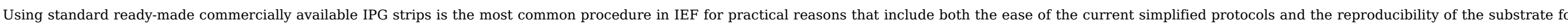

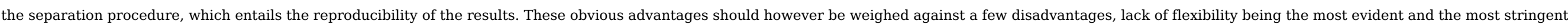

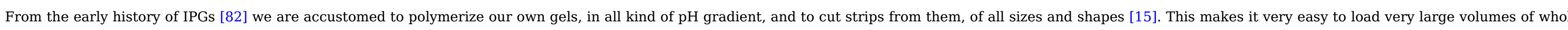

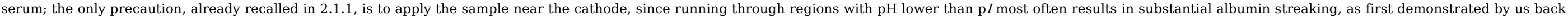

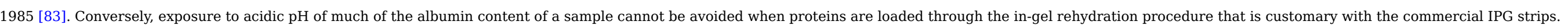

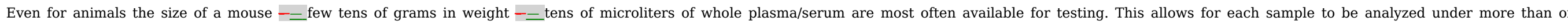

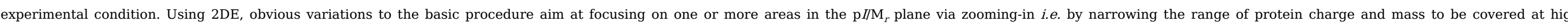

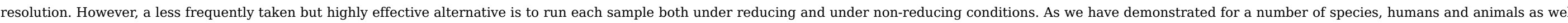

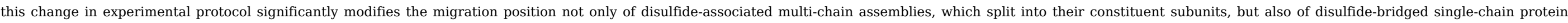
whose unfolding in the presence of SDS is restricted by the presence of covalent bonds [84]

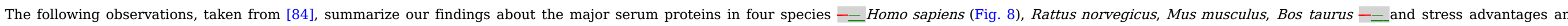

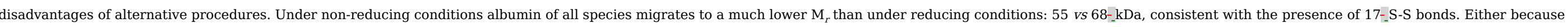

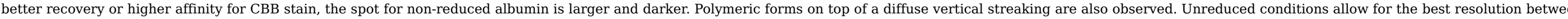

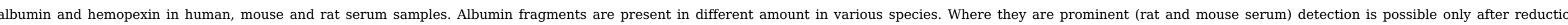
- = which implies the presence in rat and mouse circulation of a substantial amount of cleaved but undissociated albumin.

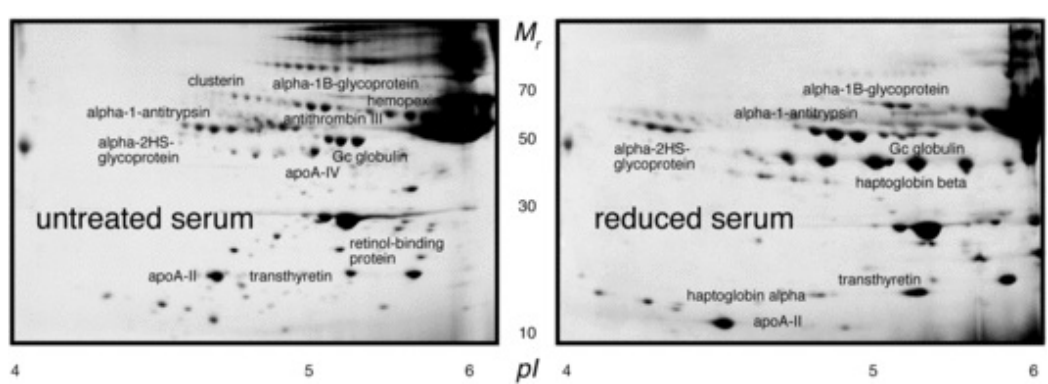

Fig. 8 2DE pattern of serum from a control subject run under non reducing (left) vs reducing conditions (right). Our unpublished results.

alt-text: Fig. 8

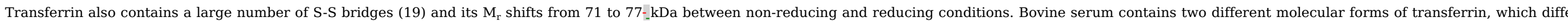

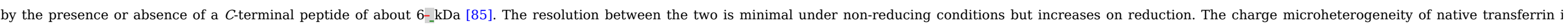

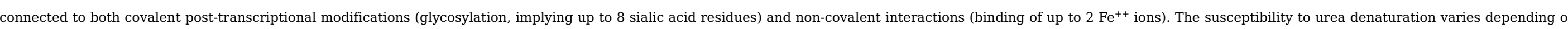

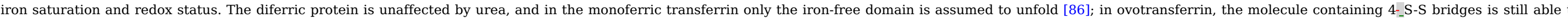

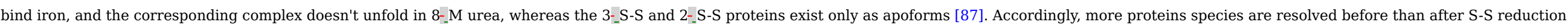

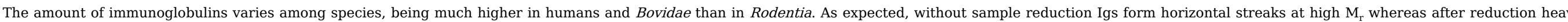
and light chains are resolved at lower $\mathrm{M}_{\mathrm{r}}$. 


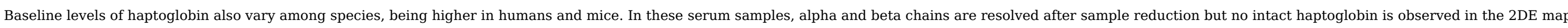

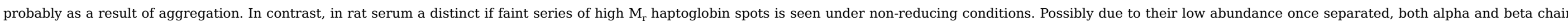

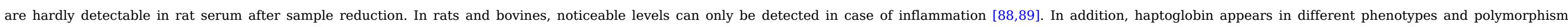
depending on the species [90].

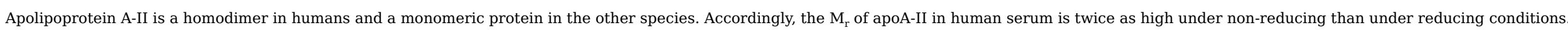

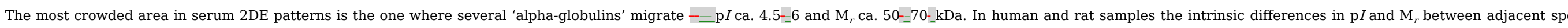

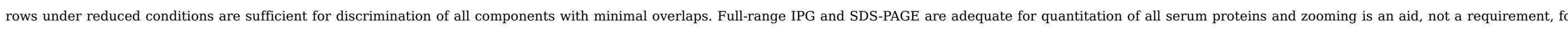

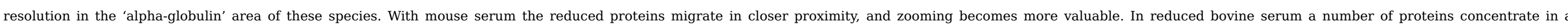

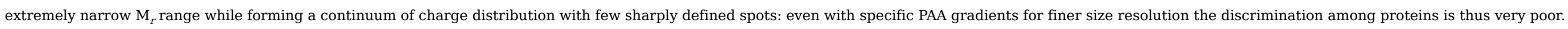

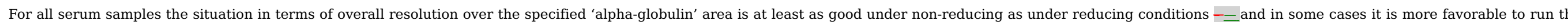

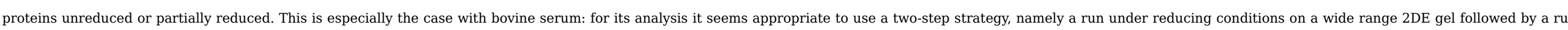

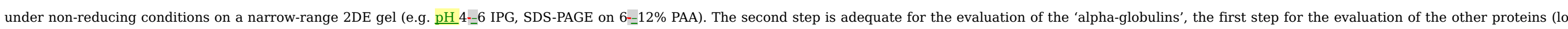
$\mathrm{M}_{r}$, or alkaline $\mathrm{p}$ ).

\section{4 Focus on major plasma/serum proteins}

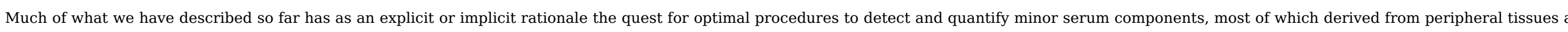
a result of pathological events. In this perspective, major serum proteins are regarded as a worthless burden. In the following we propose a different, or at least complementary, view.

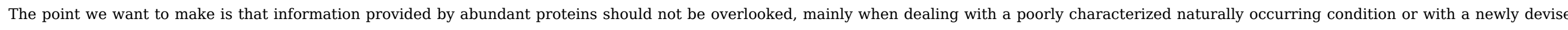

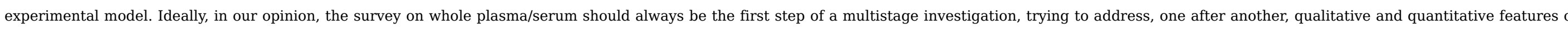
different sets of test proteins at increasing levels of resolution.

\subsubsection{Protein species}

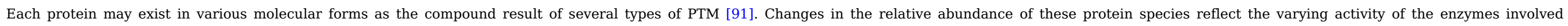

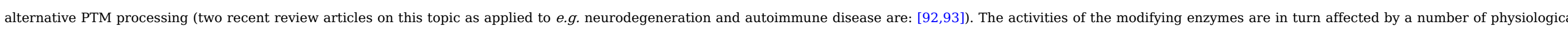
and pathological factors: data from the assessment of individual protein species may thus provide clues to the attendant conditions.

\subsection{Proteolysis}

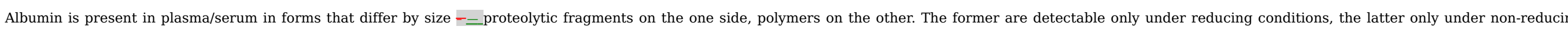

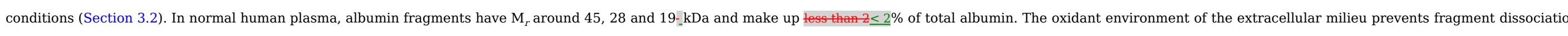
to occur in vivo: indeed, the ratio fragments vs intact albumin is not higher in urine than in plasma/serum samples from the same subject.

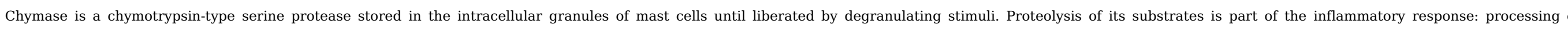

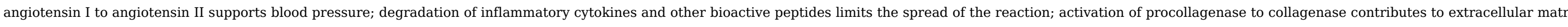

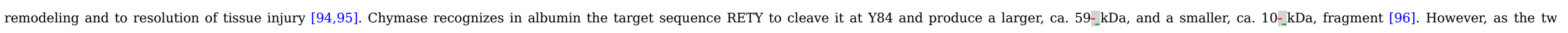
fragments do not dissociate (because of the $\mathrm{C} 75-$ 


$$
\text { - }
$$

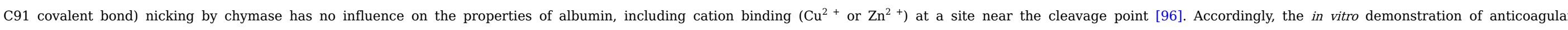
properties for $S$-carboxymethylated CNBr-fragments of albumin is unlikely to have any in vivo counterpart, and not only because of the unphysiological type of cleavage [97].

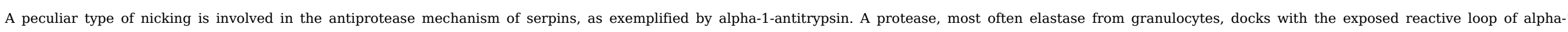

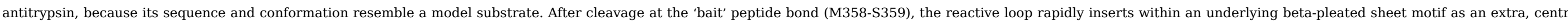

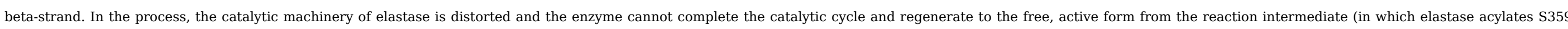

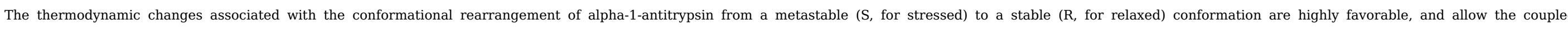

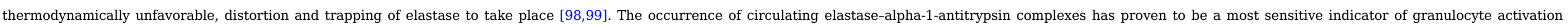

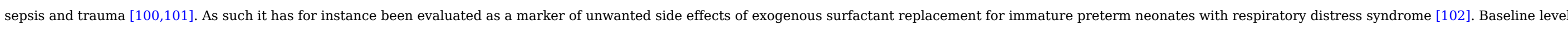
of the complexes are around 0.1- $\mu \mathrm{g} / \mathrm{mL}$ to increase a few folds during granulocyte activation [103]; their quantitation is made by ELISA.

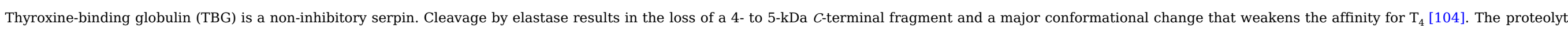

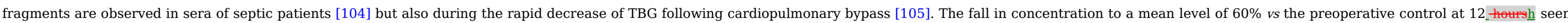
thus connected with the local inflammatory response to the surgical procedure and results in the accumulation of free $\mathrm{T}_{4}$ at the inflamed sites [105].

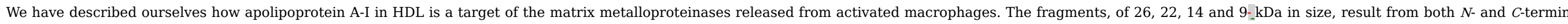

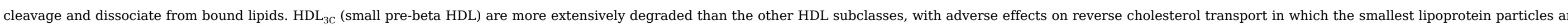

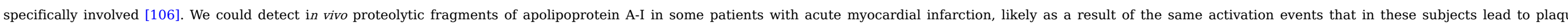

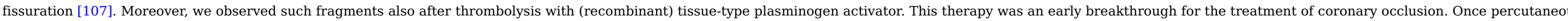

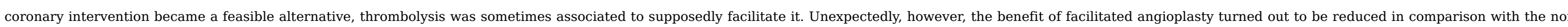
facilitated intervention. Degradation of circulating HDL may provide a potential mechanism for this outcome.

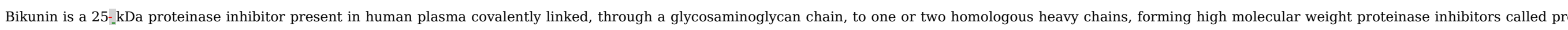

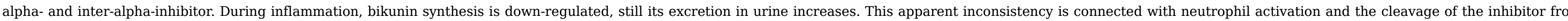
the $C$-terminus of the heavy chains by the released proteases [108].

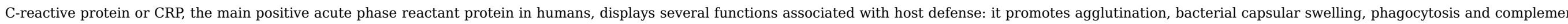

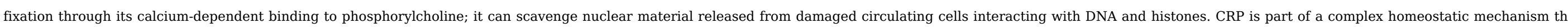

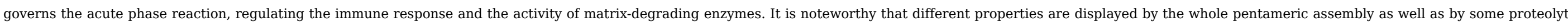

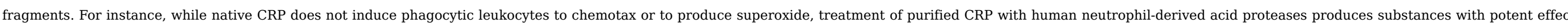

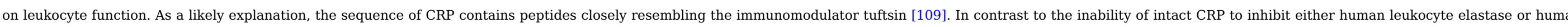

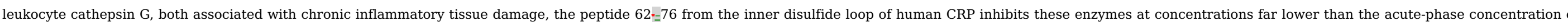

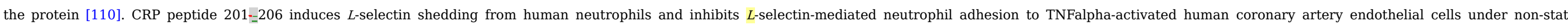
conditions. It also attenuates shear-induced up-regulation of platelet $P$-selectin expression, platelet capture of neutrophils, and subsequent homotypic neutrophil adhesion in human whole blood [111].

\subsection{Polymerization}

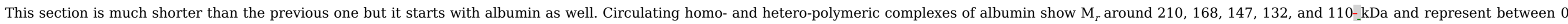

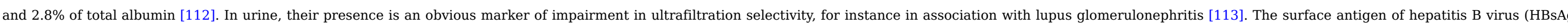
acts as a receptor for polymerized human serum albumin; its levels are predictive of the outcome of the disease [114,115],

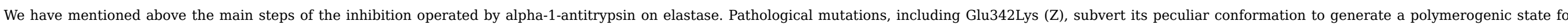




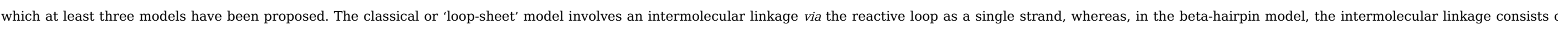

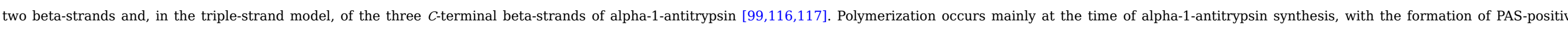

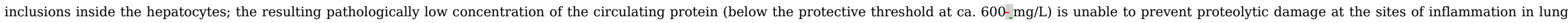

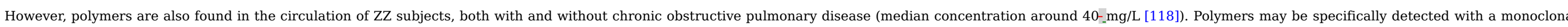
antibody that recognizes a conformation-dependent neoepitope on both polymerized and elastase-complexed molecular forms of alpha-1-antitrypsin [119].

\subsection{Differential glycosylation}

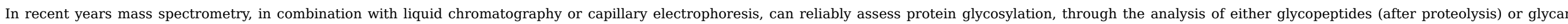

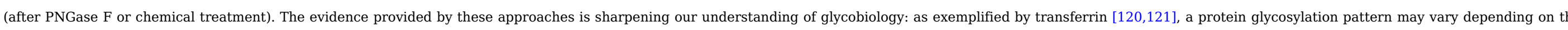
tissue in which it is synthesized or the biological fluid in which it is accumulated, and is extensively affected by different physiological and pathological states.

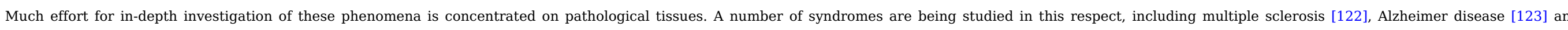

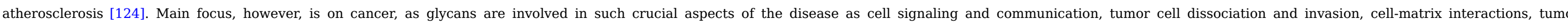
angiogenesis, immune modulation and metastasis formation [125-129].

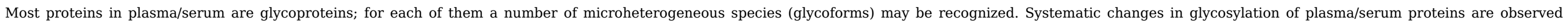

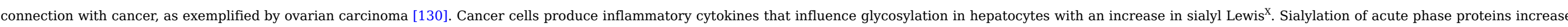

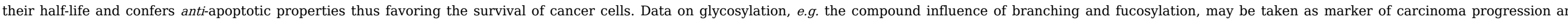

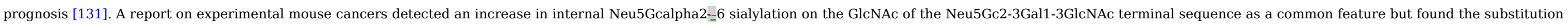

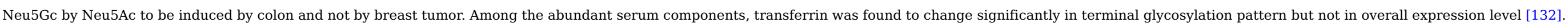

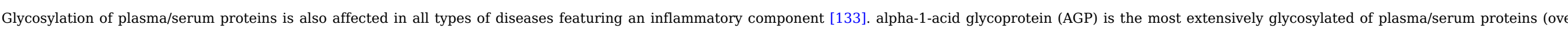

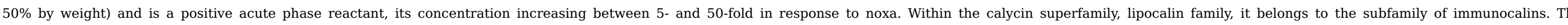

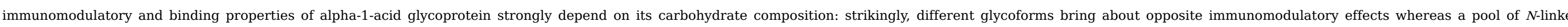

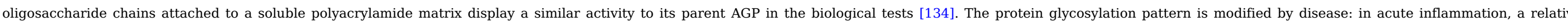

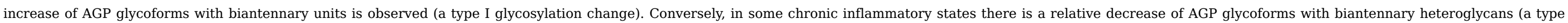

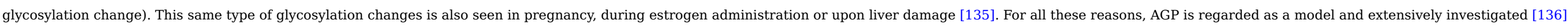

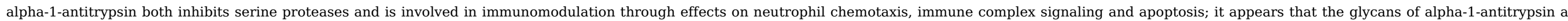
more important for the latter functions rather than for the former [137].

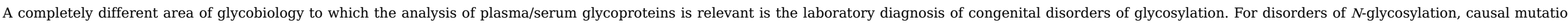

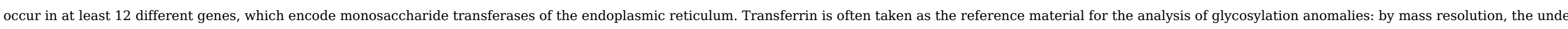
glycosylation of transferrin is characterized as the total absence of one or both $N$-linked oligosaccharides [138].

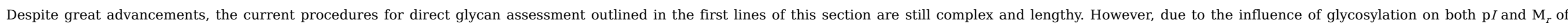

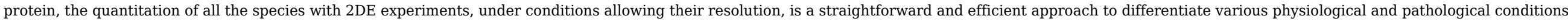

\subsubsection{Inflammation markers}

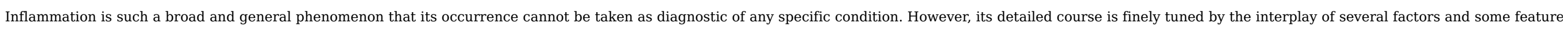
vary from one case to another.

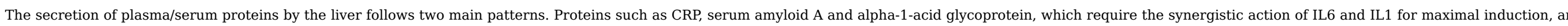




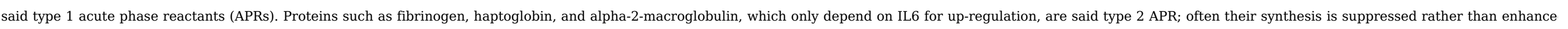

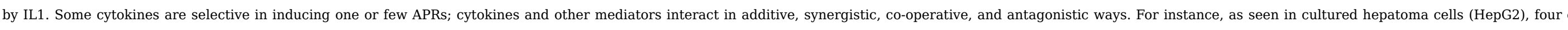

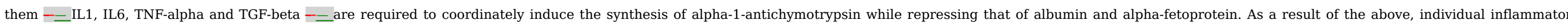
proteins differ in time-course (rapid- vs slow-reacting APRs) as well as in direction and extent of change in their circulating concentration (major positive vs moderate positive vs negative APRs).

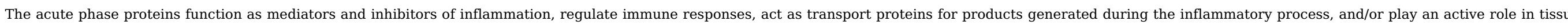

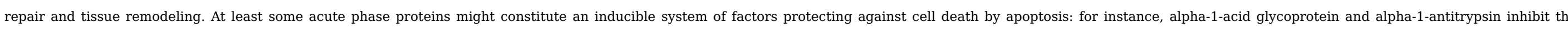
activation of caspase-3 and caspase-7 to become the main effectors (executioners) of apoptosis [139].

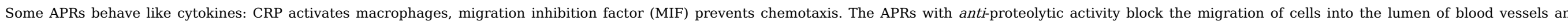
prevent the establishment of a generalized systemic inflammation.

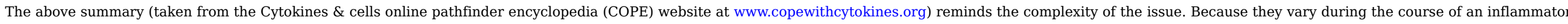

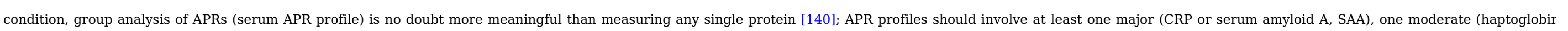

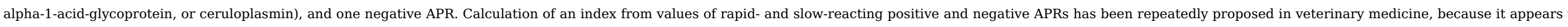
increase statistical sensitivity and specificity for detecting non-healthy subjects [141].

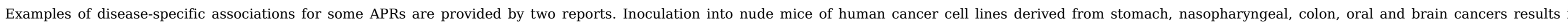

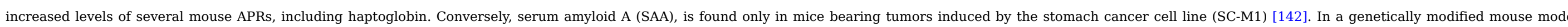

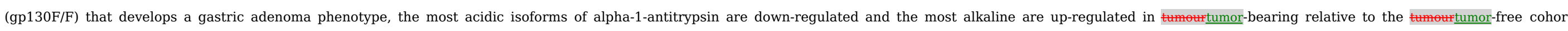
$[143,144]-=$ this latter case (Fig. 9) being connected as well to the issue of differential glycosylation in Section 4.1.3.

isoform-specific regulation in gastric cancer

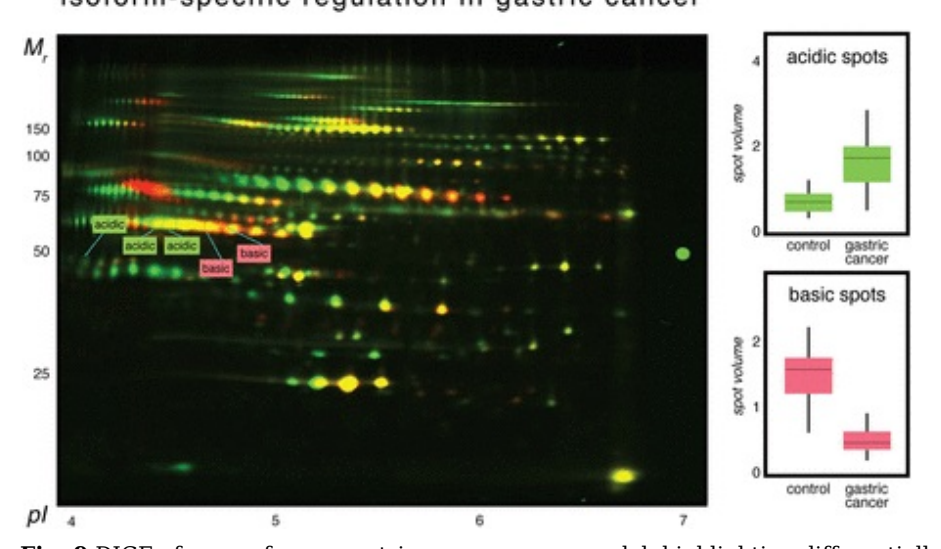

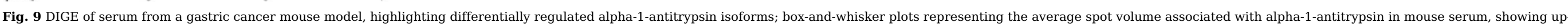
regulation (top panel, acid spots) and down-regulation (bottom panel, basic spots) in thertumor-bearing relative to the cohort. (From [144]). alt-text: Fig. 9

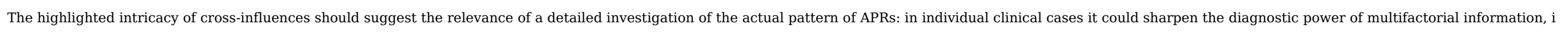

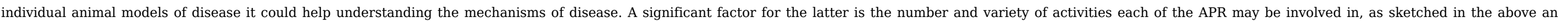
specified in the following.

\subsubsection{Moonlighting proteins?}




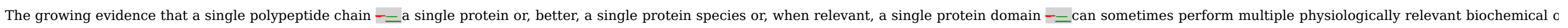

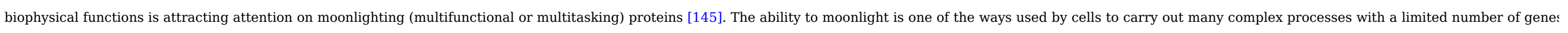

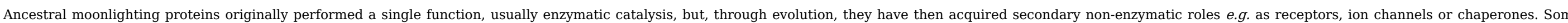

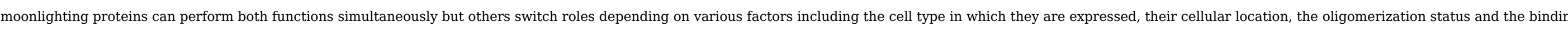

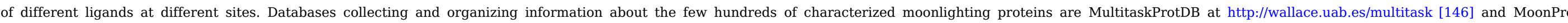
http://www.moonlightingproteins.org [147]. First attempts have started towards the computational characterization of moonlighting proteins [148] and their identification via bioinformatics tools [149].

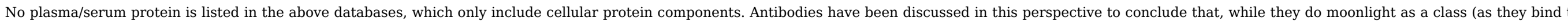

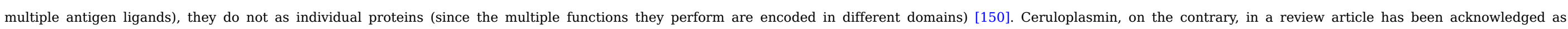

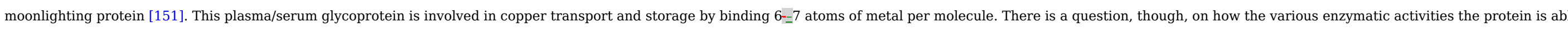

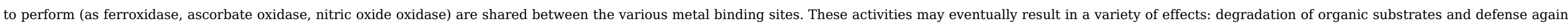
oxidant stress and overall anti-oxidant(split word: oxi-dant (see pdf)) activity or, conversely, under peculiar circumstances, pro-oxidant activity.

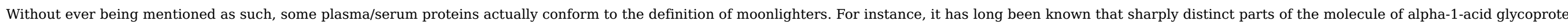

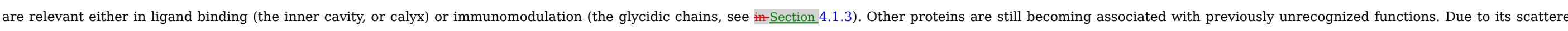
nature, relevant literature is not easily searched for by keywords; the two examples in the following paragraphs should be taken as anecdotal rather than exhaustive on recent advances.

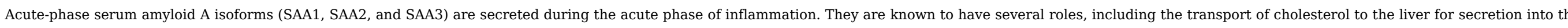

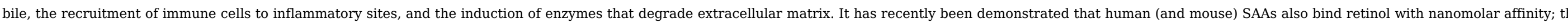

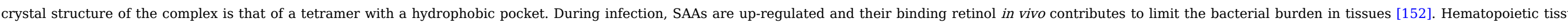

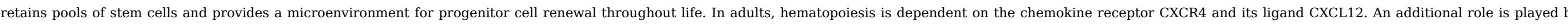

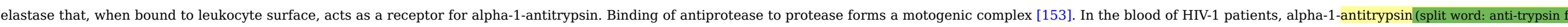
line-break (see pdf)) is bound and inactivated by anti-HIV-1 gp120 antibodies; the presence of such immune complexes correlates with the decrease in number of CD4+ lymphocytes in these subjects [154].

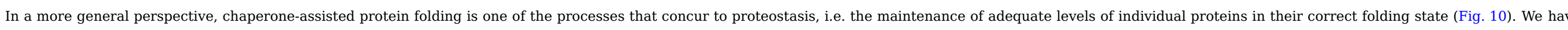

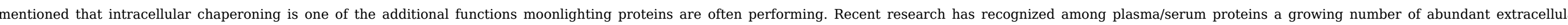

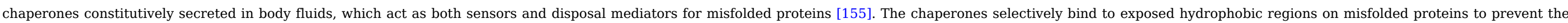

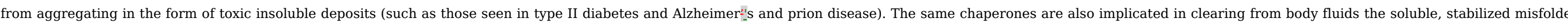
proteins via receptor-mediated endocytosis for subsequent lysosomal degradation [156].

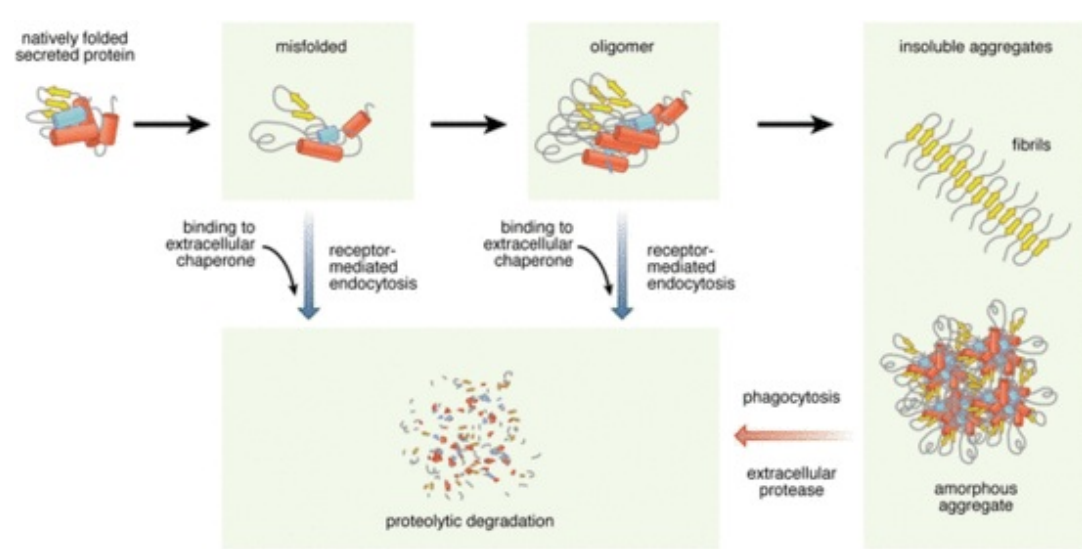




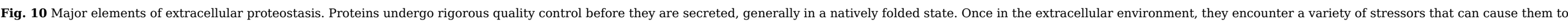

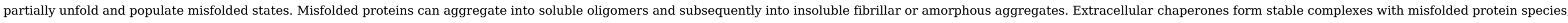

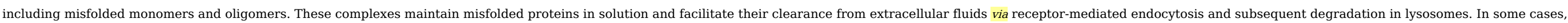

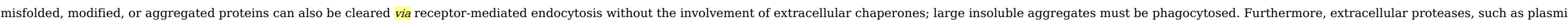
may be activated by protein aggregates and subsequently degrade them. (From [155]).

\section{alt-text: Fig. 10}

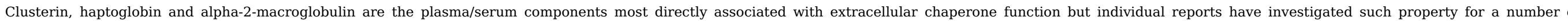

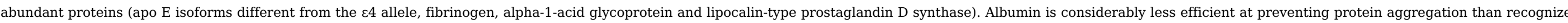
chaperones but, given its abundance, its activity may be physiologically relevant (e.g. to prevent amyloid formation by Abeta and by the Val30Met mutant of transthyretin).

\subsubsection{Cross-regulation}

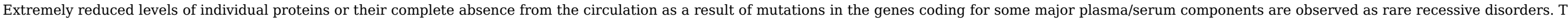

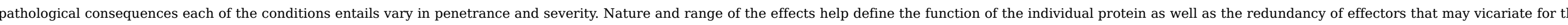

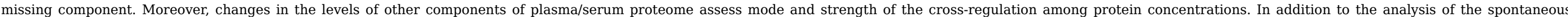
occurring null phenotypes in the human population, these aspects of proteostasis might be studied in such animal models as the knock-out (KO) mice.

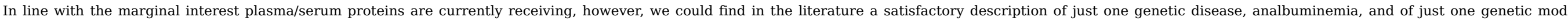

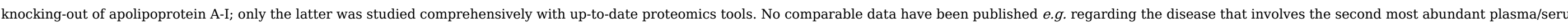

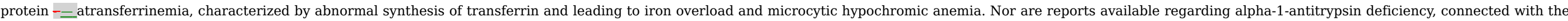

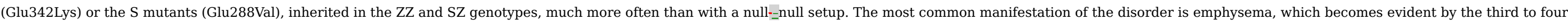

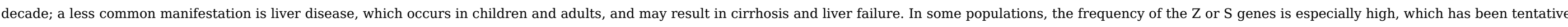
connected with heterozygote advantage against lung infections.

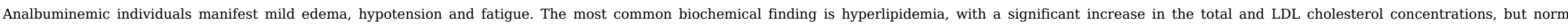

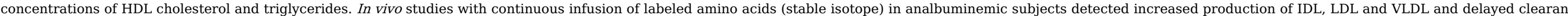

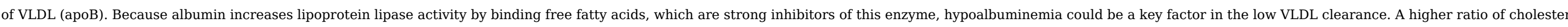

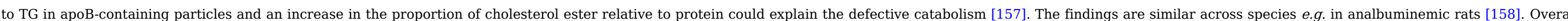

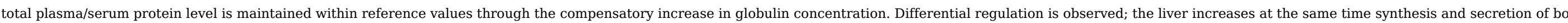

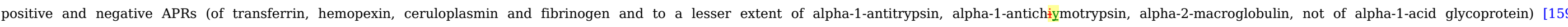

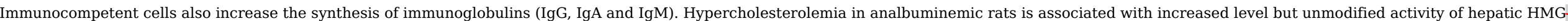

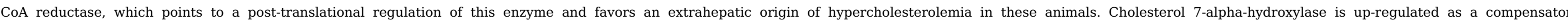
response to hypercholesterolemia [160].

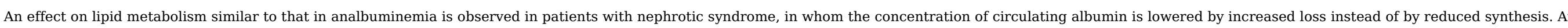

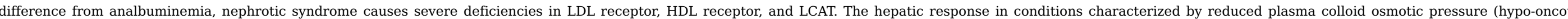

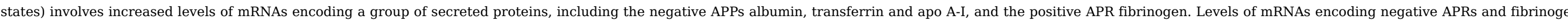
correlate with one another, suggesting that they are coordinately controlled. The transcription factors EGRF-1 and HNF-4 seem to be involved in this regulation [161].

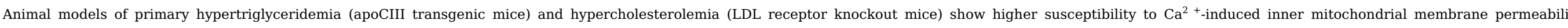

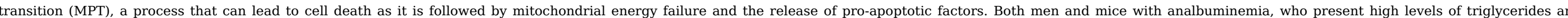




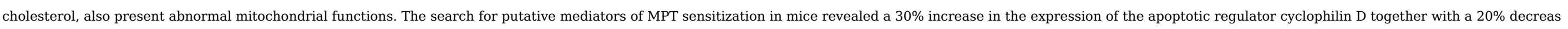
in mitochondrial nitrosothiol content: nearly $80 \%$ of total plasma reduced thiol groups and nitrosothiols reside in albumin molecules [162] :

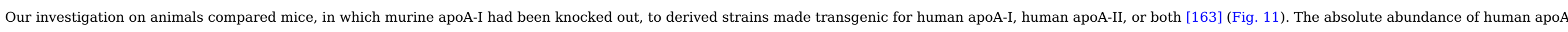

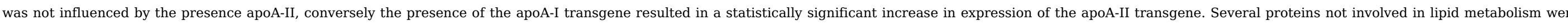

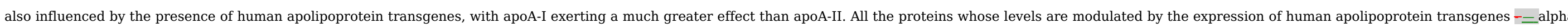

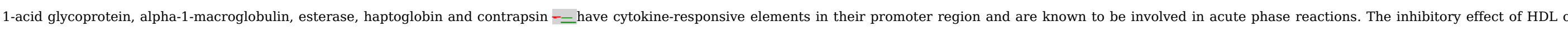
cytokine secretion [164-166] could be the explanation for our observations on the effect of expression of apolipoprotein transgenes.
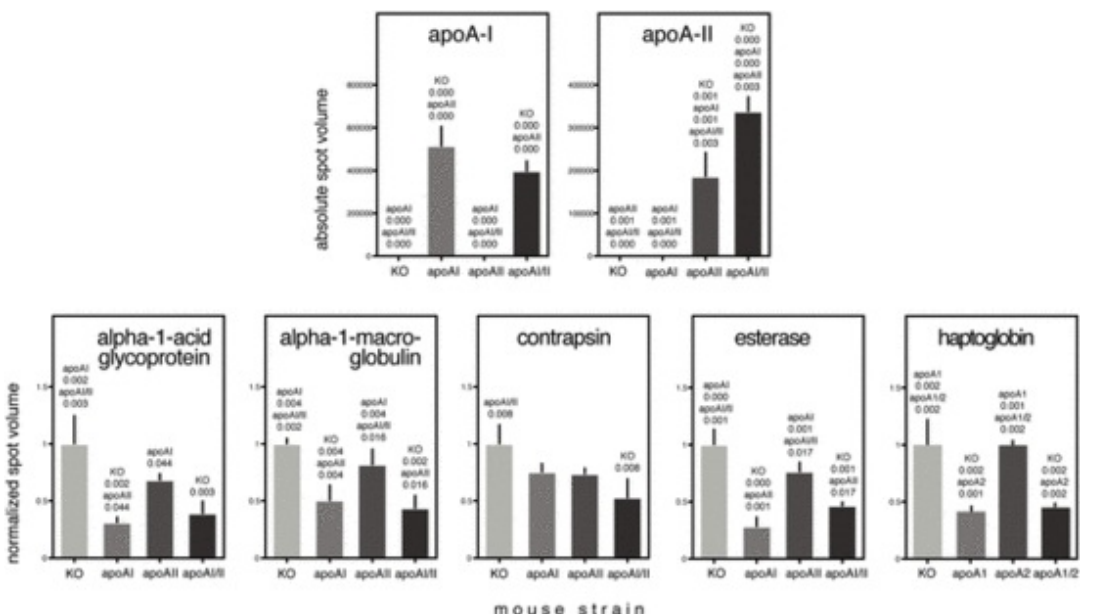

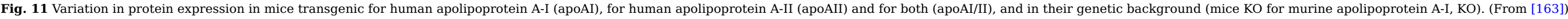
alt-text: Fig. 11

\subsubsection{Immunoglobulins}

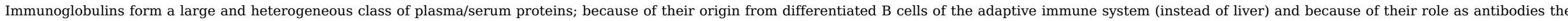
deserve a comment on their own in addition to unsystematic mention in previous sections of this review.

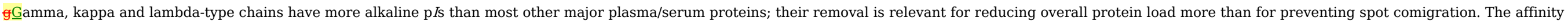

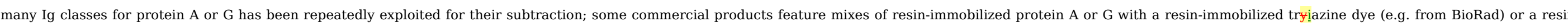

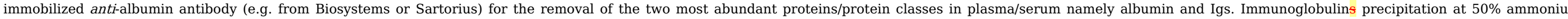
sulfate saturation is a classical procedure in the production of reagent antibodies, at both laboratory and industry scale [167].

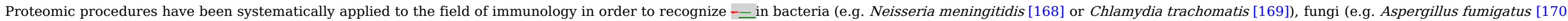

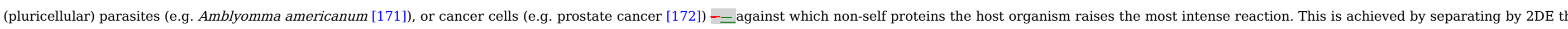

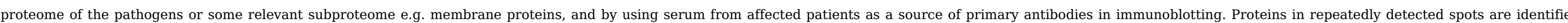

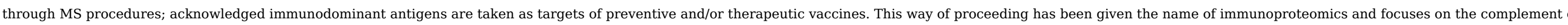
all identified antigens in a pathogen [173]. Alternative experimental approaches to electrophoresis imply the use of protein arrays of the pathogen.

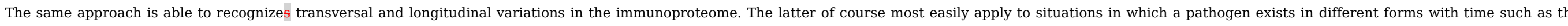

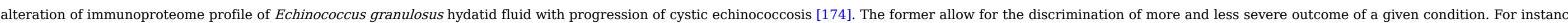




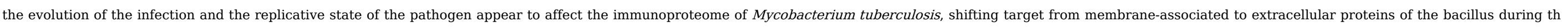

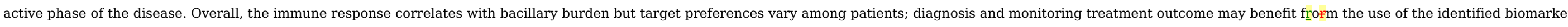

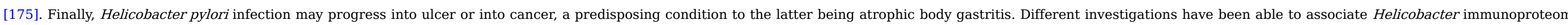

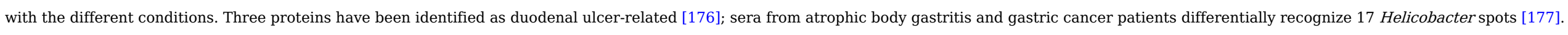

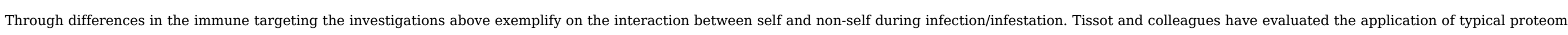

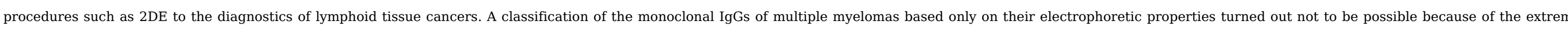

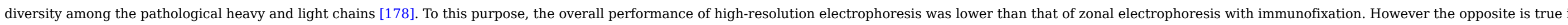
selected cases, for instance with the characterization of rare IgD myeloma [179] or with the classification of cryoglobulins [180].

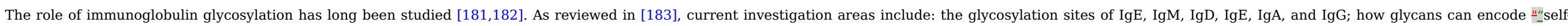

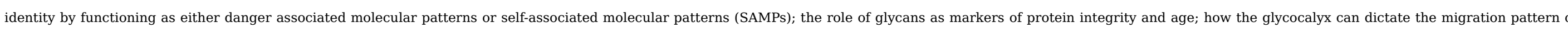

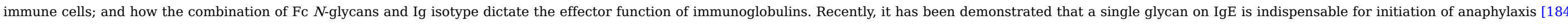

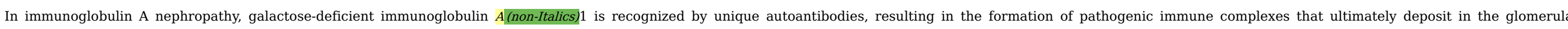

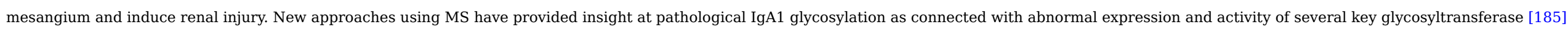

\section{5. $\underline{5}$ Conclusions}

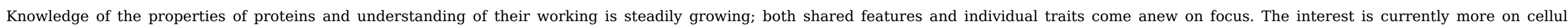

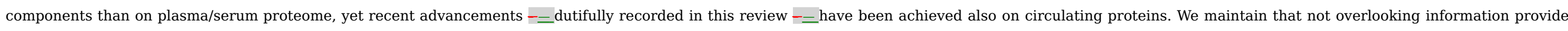
by quali- and quantitative changes in major serum/plasma proteins may effectively contribute to our awareness of the cross-influences between various levels of integration in the organism.

Supplementary data to this article can be found online at http://dx.doi.org/10.1016/j.jprot.2016.04.002.

\section{Acknowledgments}

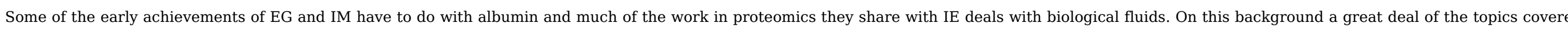
by this review are linked to our day-by-day experience more than to an assessment of the literature.

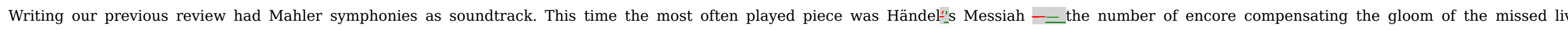
performance. But, since our interest for music is all but narrow, the title of the review positively comes from a much more recent composition.

\section{Bibliographyeferences (General comment: on several pages (in the pdf view) the two text columns are not of equal} length, e.g. pages $2,4,8,12,13 \ldots$, not always for obvious reasons.)

[1] NEN.L. Anderson and NGN.G. Anderson, The human plasma proteome: history, character, and diagnostic prospects, Mol. Cell. Proteomics 1, $2002,845-\underline{\underline{6}} 67$.

[2] S. Subramanian, Dye-ligand affinity chromatography: the interaction of Cibacron Bblue F3GA with proteins and enzymes, CRC Crit. Rev. Bioeng. 16, $1984,169-205$.

[3] AA. Denizli and EE. Piskin, Dye-ligand affinity systems, J. Biochem. Biophys. Methods 49, 2001, 391-416.

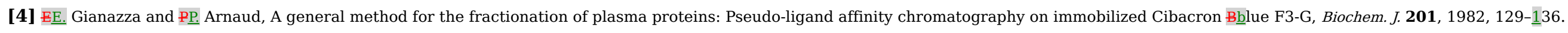

[5] EEE. Gianazza and P. Arnaud, Chromatography of plasma proteins on immobilized Cibacron Bblue F3-GA. Mechanism of the molecular interaction, Biochem. J. 203, 1982, 637- $\underline{6} 41$.

[6] 프. Miller and AM. Gemeiner, An electrophoretic study on interactions of albumins of different species with immobilized Cibacron Bblue F3G A, Electrophoresis 19, 1998, 2506-2514.

[7] FE. Di Girolamo and PGP.G. Righetti, Plasma proteomics for biomarker discovery: a study in blue, Electrophoresis 32, 2011, 3638- $\underline{36} 44$. 


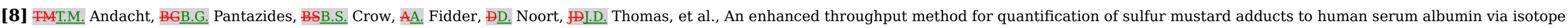
dilution tandem mass spectrometry, J. Anal. Toxicol. 38, 2014, 8-15.

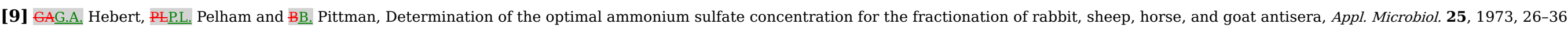

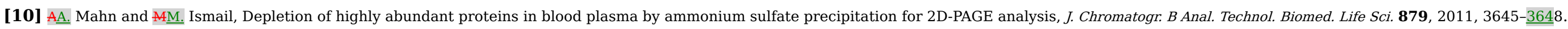

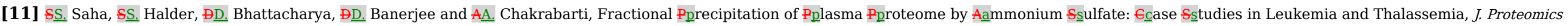
Bioinform. 5, 2012, 163-171.

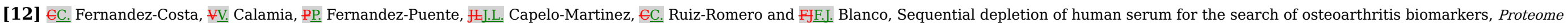
Sci. 10, 2012, 55

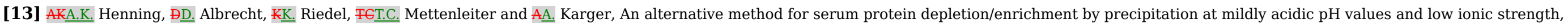
Proteomics 15, 2015, 1935-1940.

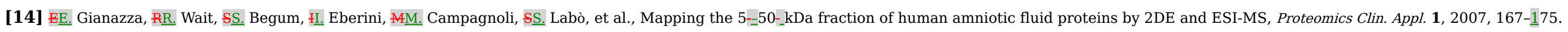

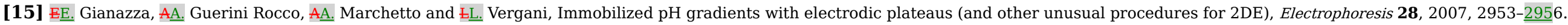

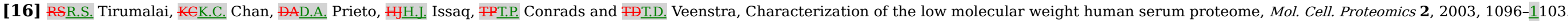

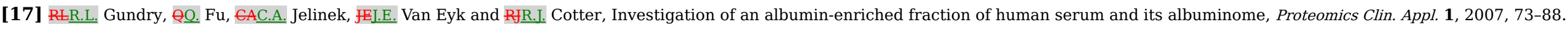

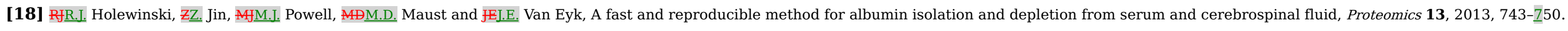

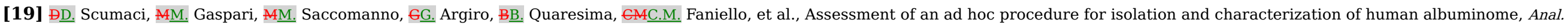
Biochem. 418, 2011, 161- $\underline{\underline{16} 3 .}$

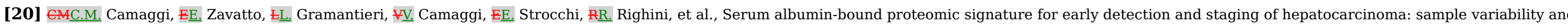
data classification, Clin. Chem. Lab. Med. 48, 2010, 1319-1326.

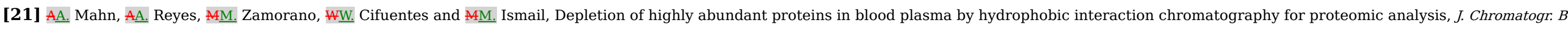
Anal. Technol. Biomed. Life Sci. 878, 2010, 1038-1044.

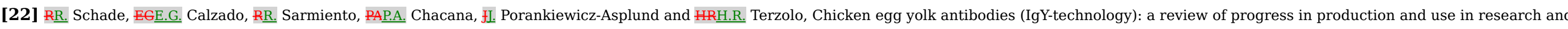
human and veterinary medicine, Altem. Lab. Anm.Altern. Lab. Anim 33, 2005, 129-154.

[23] SHS.H. Tan, AA. Mohamedali, AA. Kapur and MSM.S. Baker, Ultradepletion of human plasma using chicken antibodies: a proof of concept study, J. Proteome Res. 12, 2013, 2399-2 413.

[24] MMM.M. Harmsen and Hy.J. De Haard, Properties, production, and applications of camelid single-domain antibody fragments, Appl. Microbiol. Biotechnol. 77, 2007, 13-22.

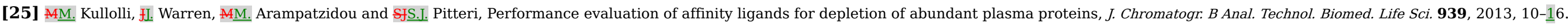

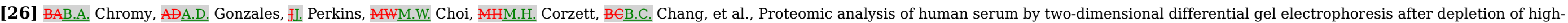
abundant proteins, J. Proteome Res. 3, 2004, 1120-1127.

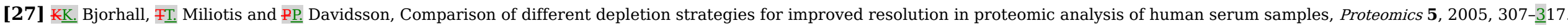

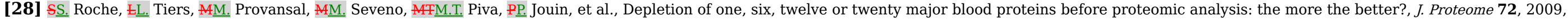


$945-\underline{9} 51$.

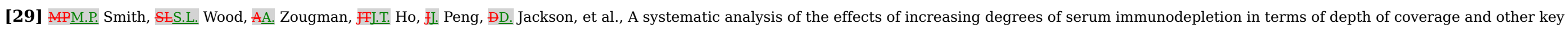
aspects in top-down and bottom-up proteomic analyses, Proteomics 11, 2011, 2222-22 35.

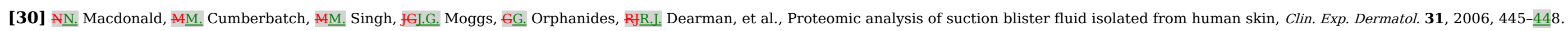

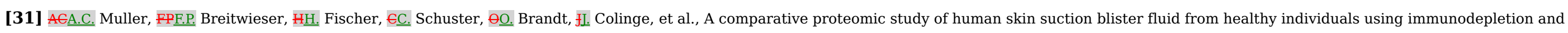
iTRAQ labeling, J. Proteome Res. 11, 2012, 3715- $\underline{\underline{37}} 27$.

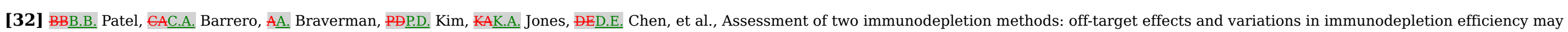
confound plasma proteomics, J. Proteome Res. 11, 2012, 5947- $\underline{\underline{59} 58 .}$

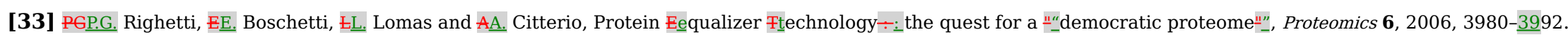

[34] PGP.G. Righetti, EE. Boschetti and GG. Candiano, Mark Twain: how to fathom the depth of your pet proteome, J. Proteome 75, $2012,4783-\underline{4791 .}$

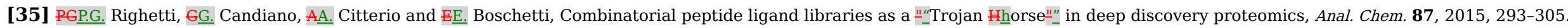

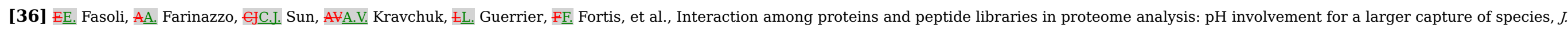
Proteome 73, 2010, 733- $\underline{7} 42$.

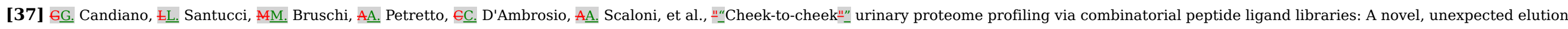
system, J. Proteome 75, 2012, 796-805.

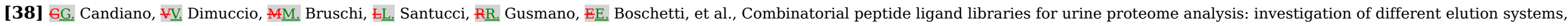
Electrophoresis 30, 2009, 2405-2411.

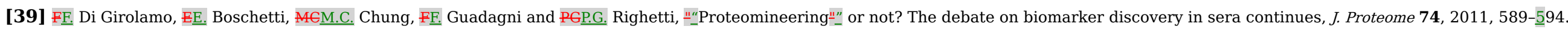

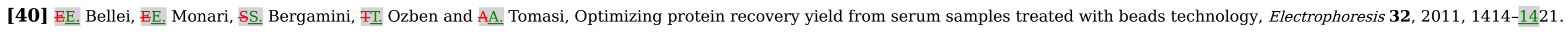

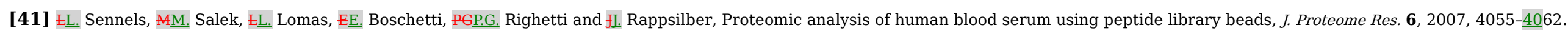

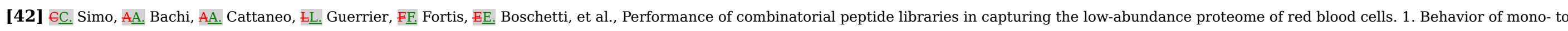
hexapeptides, Anal. Chem. 80, 2008, 3547- $\underline{35} 56$.

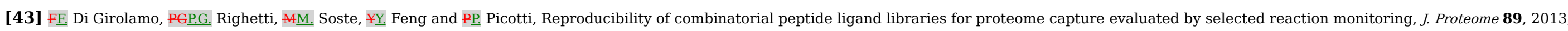
215-226.

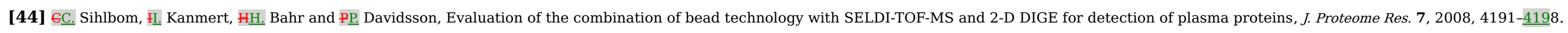

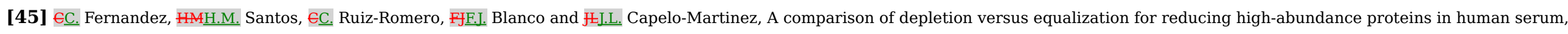
Electrophoresis 32, 2011, 2966-2974.

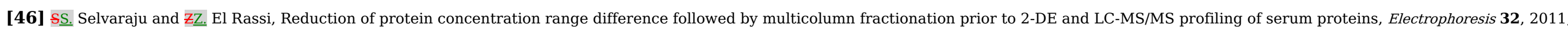
674- $\underline{6} 85$.

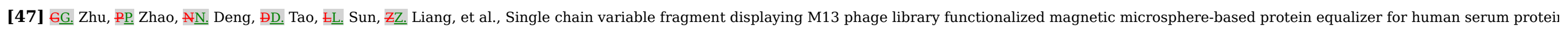
analysis, Anal. Chem. 84, 2012, 7633-7637. 


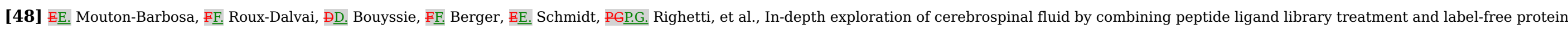
quantification, Mol. Cell. Proteomics 9, 2010, 1006- $\underline{\underline{10}} 21$.

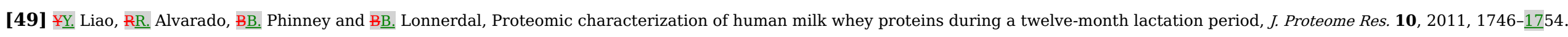

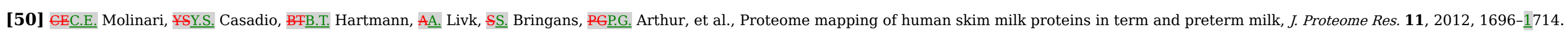

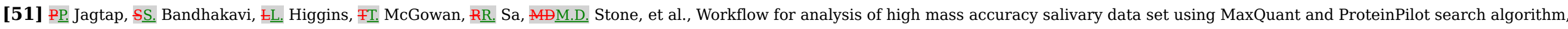
Proteomics 12, 2012, 1726-1730.

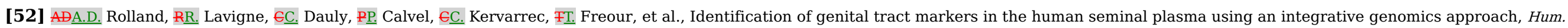
Reprod. 28, 2013, 199-209.

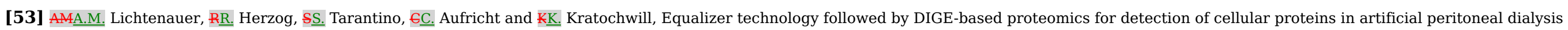
effluents, Electrophoresis 35, 2014, 1387-1394.

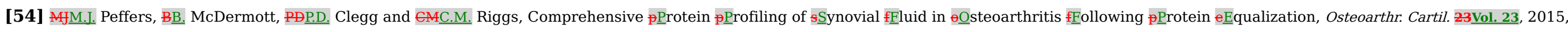
Osteoarthritis Research Society, 1204-1213.

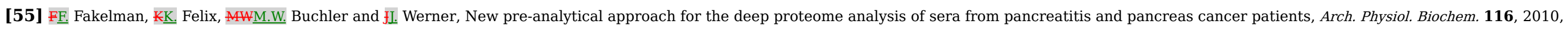
208-217.

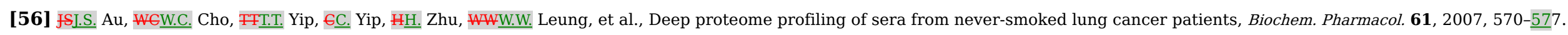

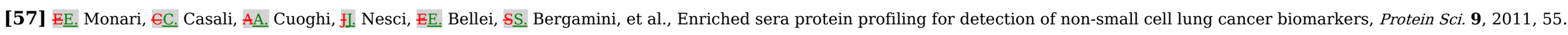

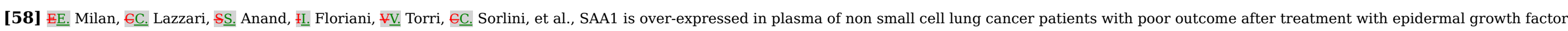
receptor tyrosine-kinase inhibitors, J. Proteome 76, 2012, 91-101, Spec No..

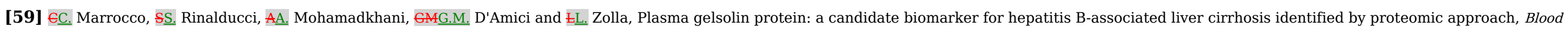
Transfus. 8 Suppl. 3, 2010, s105-s112.

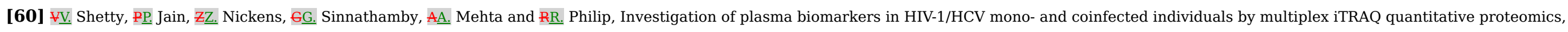
OMICS 15, 2011, 705-픅.

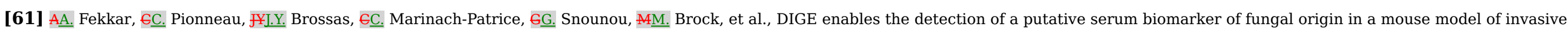
aspergillosis, J. Proteome 75, 2012, 2536-2549.

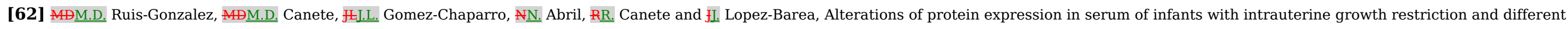
gestational ages, J. Proteome 119, 2015, 169-1 82 .

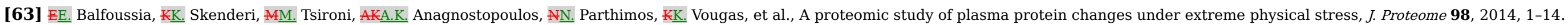

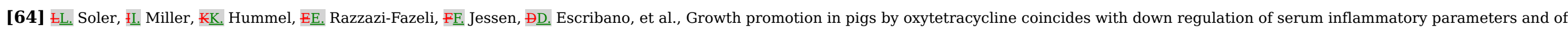
hibernation-associated protein HP-27, Electrophoresis 2016.

[65] APA.P. Drabovich, EE. Martinez-Morillo and EPE.P. Diamandis, Toward an integrated pipeline for protein biomarker development, Biochim. Biophys. Acta 2015, $1854,677-\underline{6} 86$.

[66] S. Hanash, HUPO initiatives relevant to clinical proteomics, Mol. Cell. Proteomics 3, 2004, 298-301. 


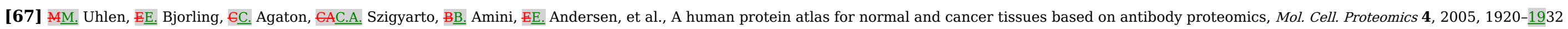

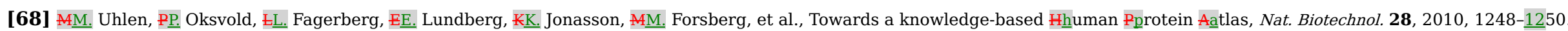

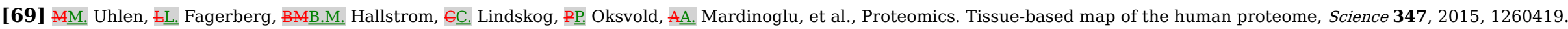

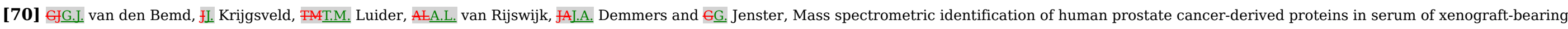
mice, Mol. Cell. Proteomics 5, 2006, 1830-1839.

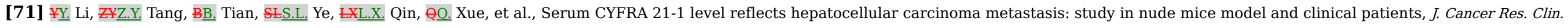
Oncol. 132, 2006, 515- $\underline{\underline{5}} 20$.

[72] ĐD. Cervi, FFT.T. Yip, NN. Bhattacharya, $\mathrm{N}$ NV.N. Podust, JI. Peterson, AA. Abou-Slaybi, et al., Platelet-associated PF-4 as a biomarker of early tumor growth, Blood 111, $2008,1201-1207$.

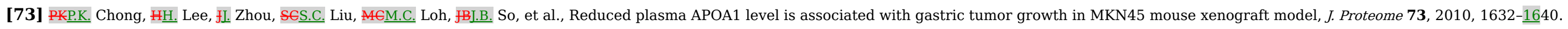

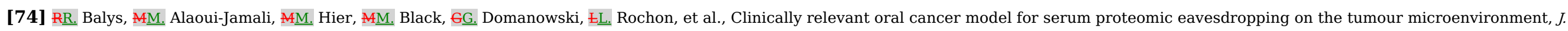
Otolaryngol. 35, 2006, 157-166.

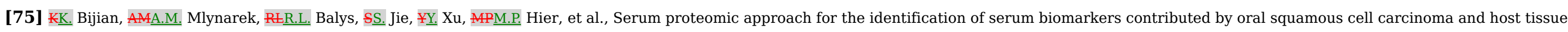
microenvironment, J. Proteome Res. 8, 2009, 2173-2185.

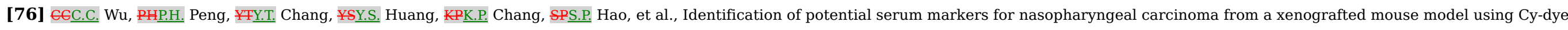
labeling combined with three-dimensional fractionation, Proteomics 8, 2008, 3605- $\underline{36} 20$.

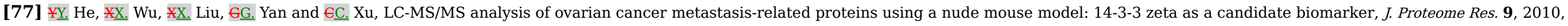
$6180-\underline{6190 .}$

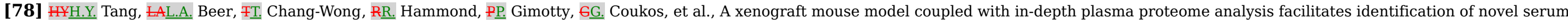
biomarkers for human ovarian cancer, J. Proteome Res. 11, 2012, 678- $\underline{6} 91$.

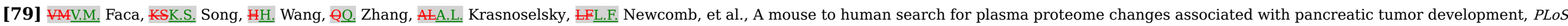
Med. 5, 2008, e123.

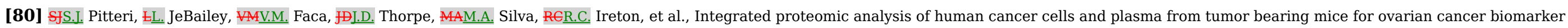
discovery, PLOS ONEPLOS One 4, 2009, e7916.

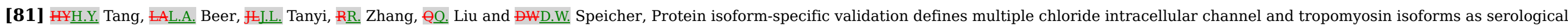
biomarkers of ovarian cancer, J. Proteome 89, 2013, 165- 178.

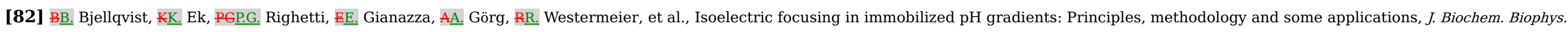
Methods 6, 1982, 317-339.

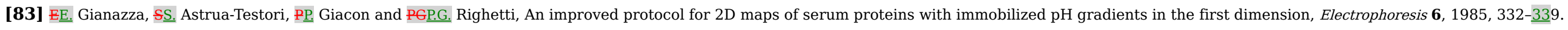

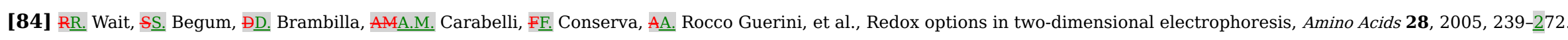

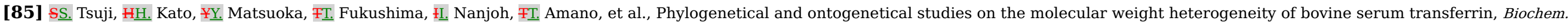
Genet. 22, 1984, 1127-1143. 
[86] RWR.W. Evans and 扛. Williams, The electrophoresis of transferrins in urea/polyacrylamide gels, Biochem. J. 189, 1980, 541- $\underline{546}$.

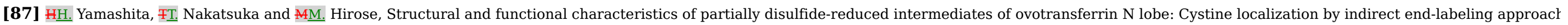
and implications for the reduction pathway, J. Biol. Chem. 270, 1995, 29806-29812.

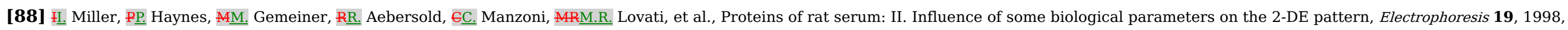
1493-1500.

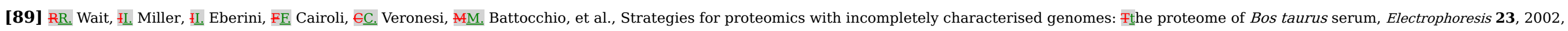
$3418-\underline{\underline{34}} 27$.

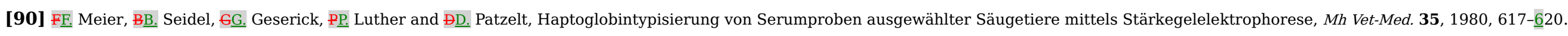

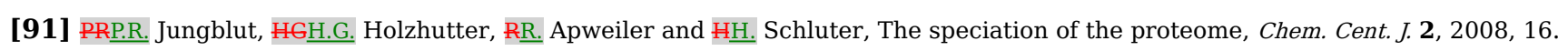

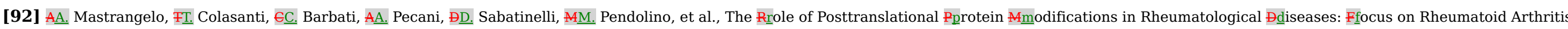
J. Immunol. Res. 2015, 2015, 712490.

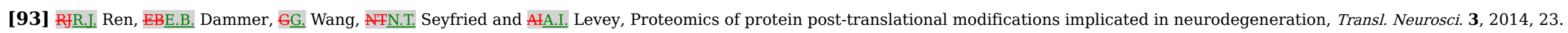

[94] 프. Fukami, 프. Okunishi and MM. Miyazaki, Chymase: its pathophysiological roles and inhibitors, Curr. Pharm. Des. 4, 1998, $439-\underline{\underline{4}} 53$.

[95] GHG.H. Caughey, Mast cell proteases as protective and inflammatory mediators, Adv. Exp. Med. Biol. 716, 2011, $212-\underline{\underline{2}} 34$.

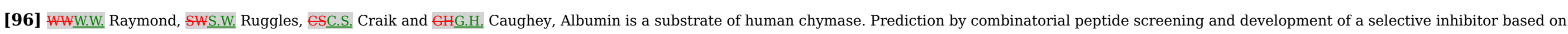
the albumin cleavage site, J. Biol. Chem. 278, 2003, 34517- $\underline{\underline{345} 24 .}$

[97] ĐKD.K. Galanakis, Anticoagulant albumin fragments that bind to fibrinogen/fibrin: possible implications, Semin. Thromb. Hemost. 18, 1992, 44-52.

[98] JAI.A. Huntington, RJR.J. Read and RWR.W. Carrell, Structure of a serpin-protease complex shows inhibition by deformation, Nature 407, 2000, 923-926.

[99] BB. Gooptu, JAI.A. Dickens and DAD.A. Lomas, The molecular and cellular pathology of alpha(1)-antitrypsin deficiency, Trends Mol. Med. 20, $2014,116-\underline{\underline{1}} 27$.

[100] EPC.P. Speer, A‥ Ninjo and MM. Gahr, Elastase-alpha 1-proteinase inhibitor in early diagnosis of neonatal septicemia, J. Pediatr. 108, 1986, 987- $\underline{9} 90$.

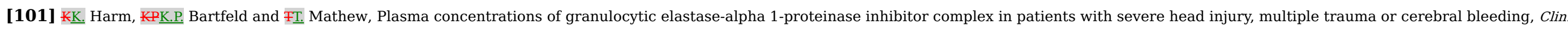
Biochem. 22, 1989, 149-153.

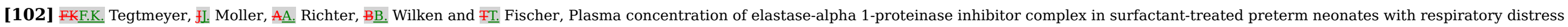
syndrome, Eur. Respir. J. 7, 1994, 260-264.

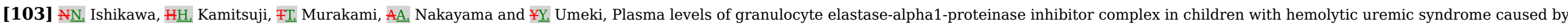
verotoxin-producing Escherichia coli, Pediatr. Int. 42, 2000, 637-641.

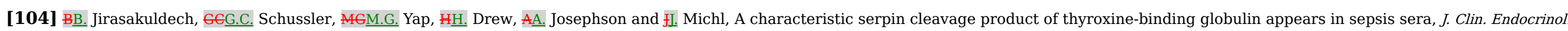
Metab. 85, 2000, 3996- $\underline{\underline{399} 9 .}$.

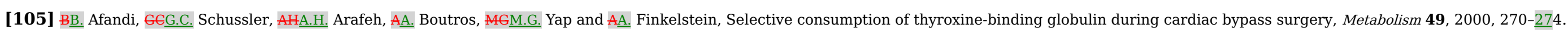

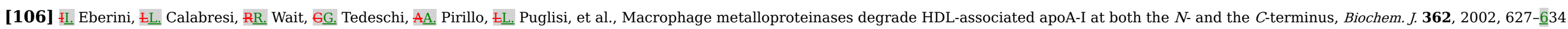




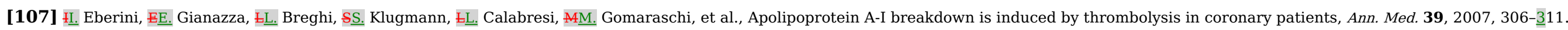

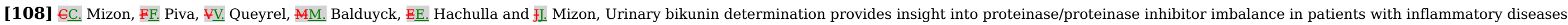
Clin. Chemistry Lab. Med. 40, 2002, 579- $\underline{\underline{5}} 86$.

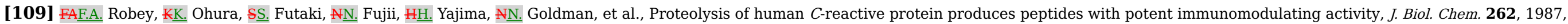
$7053-\underline{\underline{705}} 7$.

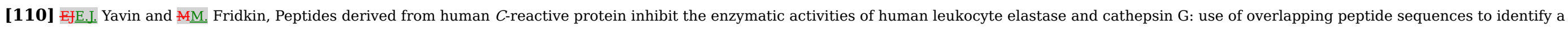
unique inhibitor, J. Pept. Res. 51, 1998, 282-28 9.

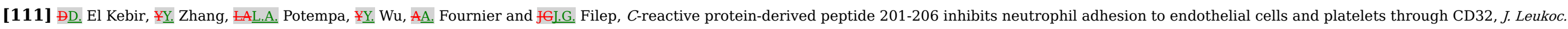
Biol. 90, 2011, 1167- $\underline{1175}$.

[112] BB. Kshirsagar, BB. Wilson and RCL.C. Wiggins, Polymeric complexes and fragments of albumin in normal human plasma, Clin. Chim. Acta 143, 1984, 265- $\underline{2} 73$.

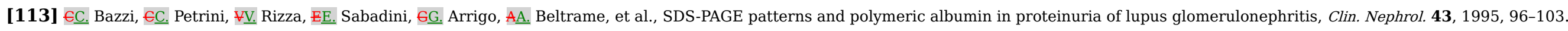

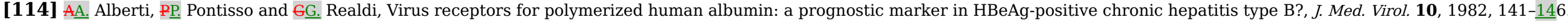

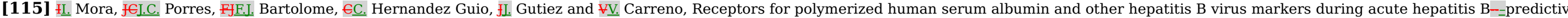
value of the outcome of the disease, Hepato-Gastroenterology 33, 1986, 250-25 4 .

[116] DAD.A. Lomas and RR. Mahadeva, Alpha1-antitrypsin polymerization and the serpinopathies: pathobiology and prospects for therapy, J. Clin. Invest. 110, 2002, 1585-1590.

[117] RWR.W. Carrell and DAD.A. Lomas, Alpha1-antitrypsin deficiency-_-a model for conformational diseases, N. Engl. J. Med. 346, 2002, 45-53.

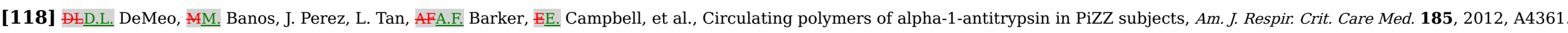

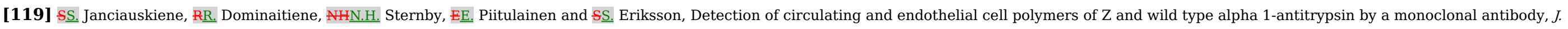
Biol. Chem. 277, 2002, 26540-26546.

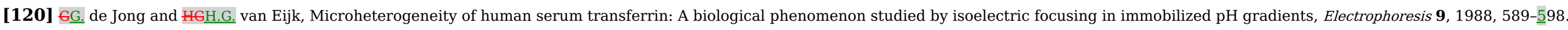

[121] GG. de Jong, WLW.L. van Noort, RAR.A. Feelders, EMC.M. de Jeu-Jaspars and HGH.G. van Eijk, Adaptation of transferrin protein and glycan synthesis, Clin. Chim. Acta 212, 1992 , 27-45.

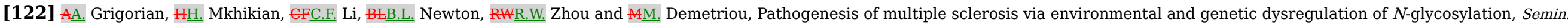
Immunopathol. 34, 2012, 415- $\underline{4} 24$.

[123] SS. Schedin-Weiss, BB. Winblad and ŁOL.O. Tjernberg, The role of protein glycosylation in Alzheimer disease, FEBS J. 281, 2014, 46-62.

[124] €Q. Pu and $€ \underline{C}$. Yu, Glycosyltransferases, glycosylation and atherosclerosis, Glycoconj. J. 31, 2014, 605- $\underline{6} 11$.

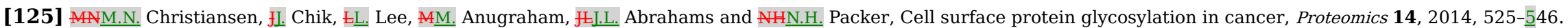

[126] ZZ. Ma and KK. Vosseller, Cancer metabolism and elevated $O$-GlcNAc in oncogenic signaling, J. Biol. Chem. 289, $2014,34457-\underline{\underline{344}} 65$.

[127] RRR.R. Drake and EL. Ball, Glycosylation and Cancer, Advances in Cancer Research 2015, Elsevier.

[128] SSS.S. Pinho and EAC.A. Reis, Glycosylation in cancer: mechanisms and clinical implications, Nat. Rev. Cancer 15, 2015, 540- $\underline{5} 55$.

[129] SRS.R. Stowell, TT. Ju and RPR.D. Cummings, Protein glycosylation in cancer, Annu. Rev. Pathol. 10, 2015, 473-510. 


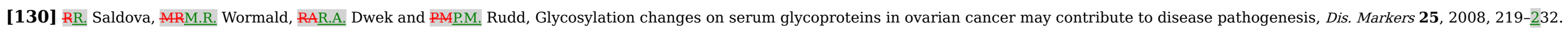

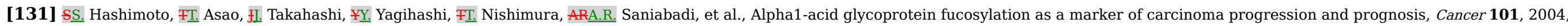
$2825-\underline{28} 36$.

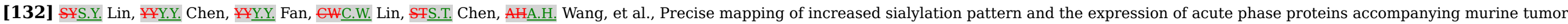
progression in BALB/c mouse by integrated sera proteomics and glycomics, J. Proteome Res. 7, 2008, 3293- 3303.

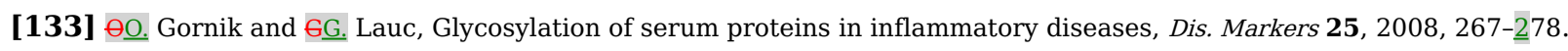

[134] SPS.D. Shiyan and AYN.V. Bovin, Carbohydrate composition and immunomodulatory activity of different glycoforms of alpha1-acid glycoprotein, Glycoconj. J. 14, 1997, 631- $\underline{638 .}$

[135] AA. Mackiewicz and KK. Mackiewicz, Glycoforms of serum alpha 1-acid glycoprotein as markers of inflammation and cancer, Glycoconj. J. 12, 1995, 241-247.

[136] FE. Ceciliani and ㅍ‥ Pocacqua, The acute phase protein alpha1-acid glycoprotein: a model for altered glycosylation during diseases, Curr. Protein Pept. Sci. 8, 2007, 91-108.

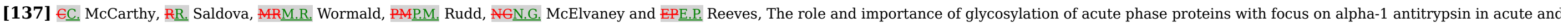
chronic inflammatory conditions, J. Proteome Res. 13, 2014, 3131- 3143.

[138] 트. Marklova and ZZ. Albahri, Screening and diagnosis of congenital disorders of glycosylation, Clin. Chim. Acta 385, 2007, 6-20.

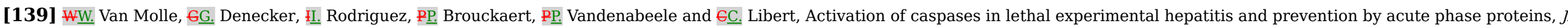
Immunol. 163, 1999, 5235- $\underline{52} 41$.

[140] PDP.D. Eckersall, Acute phase proteins as markers of inflammatory lesions, Comp. Haematol. Int. 5, 1995, 93- $\underline{9}$.

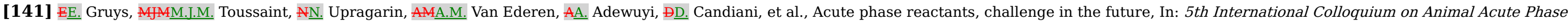
Proteins. Dublin, Ireland, 2005, 60

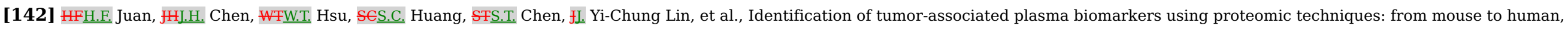
Proteomics 4, 2004, 2766-2775.

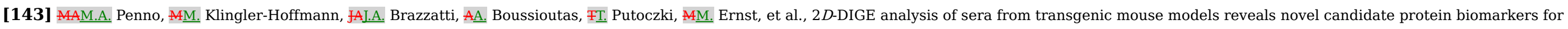
human gastric cancer, J. Proteome 77, 2012, 40-58

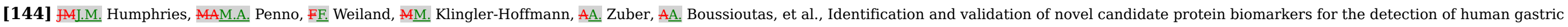
cancer, Biochim. Biophys. Acta 2014, 1844, 1051-1058.

[145] EJC.J. Jeffery, An introduction to protein moonlighting, Biochem. Soc. Trans. 42, 2014, 1679-16 83.

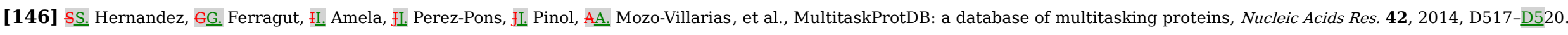

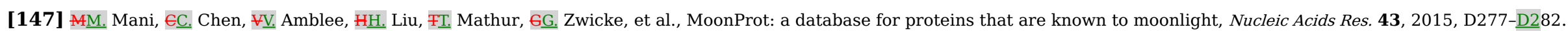

[148] $\# \underline{\text { I.K. }}$ Khan and $\boxplus$ D. Kihara, Computational characterization of moonlighting proteins, Biochem. Soc. Trans. 42, $2014,1780-\underline{1785}$.

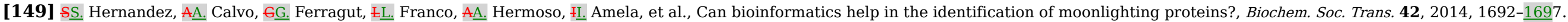

[150] AGA.C. Martin, Structural biology of moonlighting: lessons from antibodies, Biochem. Soc. Trans. 42, 2014, $1704-\underline{170} 8$.

[151] PP. Bielli and $\pm \underline{L}$. Calabrese, Structure to function relationships in ceruloplasmin: a İmoonlighting 


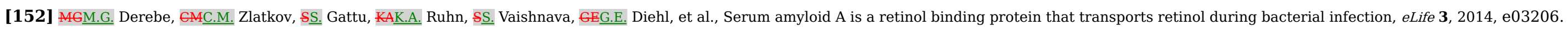

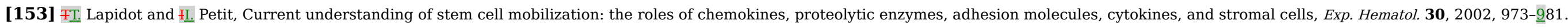

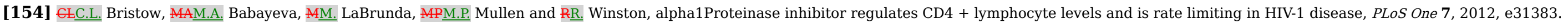

[155] ARA.R. Wyatt, JI.J. Yerbury, H $\underline{H}$. Ecroyd and MRM.R. Wilson, Extracellular chaperones and proteostasis, Annu. Rev. Biochem. 82, 2013, 295-322.

[156] ARA.R. Wyatt, JJ.J. Yerbury, RAR.A. Dabbs and MRM.R. Wilson, Roles of extracellular chaperones in amyloidosis, J. Mol. Biol. 421, 2012, $499-516$.

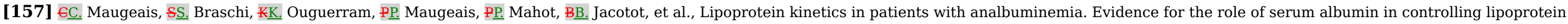
metabolism, Arterioscler. Thromb. Vasc. Biol. 17, 1997, 1369-1375.

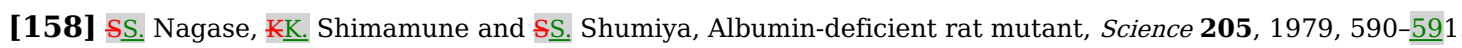

[159] KK. Sugiyama, FT. Emori and S. S. Nagase, Synthesis and secretion of plasma proteins by isolated hepatocytes of analbuminemic rats, J. Biochem. 92, 1982, 775-779.

[160] KK. Liang and NDN.D. Vaziri, HMG-CoA reductase, cholesterol 7alpha-hydroxylase, LCAT, ACAT, LDL receptor, and SRB-1 in hereditary analbuminemia, Kidney Int. 64, 2003, 192-198.

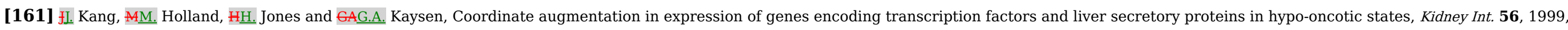
$452-\underline{4} 60$.

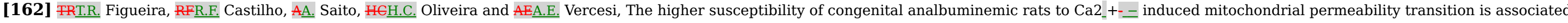
with the increased expression of cyclophilin D and nitrosothiol depletion, Mol. Genet. Metab. 104, 2011, 521- $\underline{528 .}$

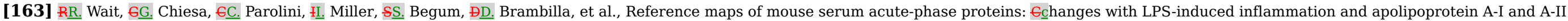
transgenes, Proteomics 5, 2005, 4245- $\underline{\underline{42} 53 .}$.

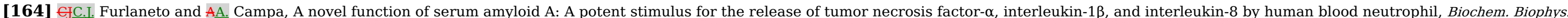
Res. Commun. 268, 2000, 405- $\underline{\underline{40} 8 .}$

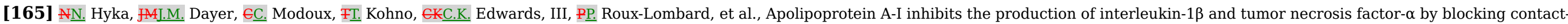
mediated activation of monocytes by T lymphocytes, Blood 97, 2001, 2381-2389.

[166] GJC.J. Furlaneto, FPE.P. Ribeiro, EE. Hatanaka, GG. Souza, MAM.A. Cassatella and AA. Campa, Apolipoproteins A-I and A-II downregulate neutrophil functions, Lipids 37, 2002, 925-928.

[167] BAEB.A.L. Hurn and SMS.M. Chantler, Production of reagent antibodies, Methods Enzymol. 70, 1980, $104-\underline{1} 41$.

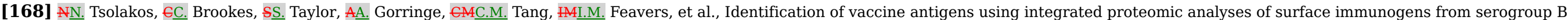
Neisseria meningitidis, J. Proteome 101, 2014, 63-76.

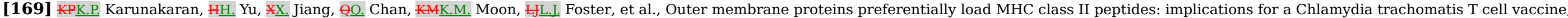
Vaccine 33, 2015, 2159-2166.

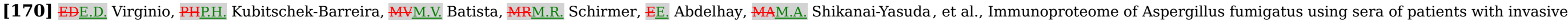
aspergillosis, Int. J. Mol. Sci. 15, 2014, 14505- $\underline{145} 30$.

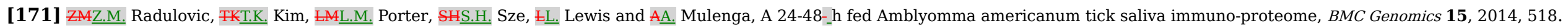

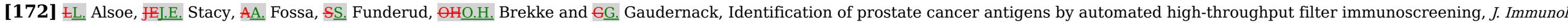
Methods 330, 2008, 12-23. 
[173] AA. Krah and PRP.R. Jungblut, Immunoproteomics, Methods Mol. Med. 94, 2004, 19-32.

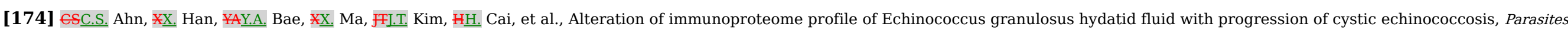
Vectors 8, 2015, 10

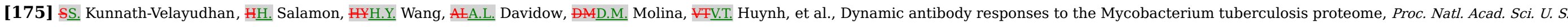
A. 107, 2010, 14703-14708.

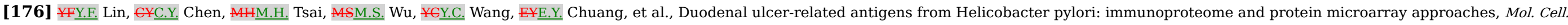
Proteomics 6, 2007, 1018- $\underline{1026}$.

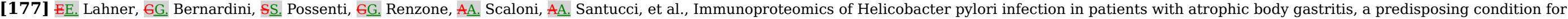
gastric cancer, Int. J. Med. Microbiol. 301, 2011, 125-1 32 .

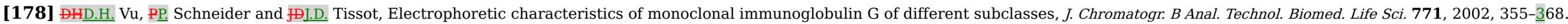

[179] JI. Stulik, $\mathrm{MM}$. Tichy and $\underline{H} \underline{\mathrm{H}}$. Kovarova, Two-dimensional gel electrophoresis of four serum samples from patients with IgD myeloma, Clin. Chim. Acta 218, 1993, 149-158.

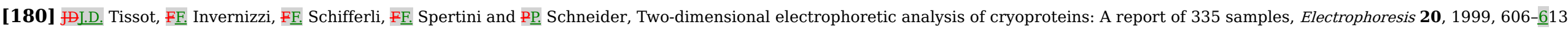

[181] H. Krotkiewski, Carbohydrate moiety of immunoglobulins in health and pathology, Acta Biochim. Pol. 46, 1999, 341- $\underline{\underline{3} 50 .}$

[182] PMP.M. Rudd, FT. Elliott, P. Cresswell, IAI.A. Wilson and RAR.A. Dwek, Glycosylation and the immune system, Science 291, 2001, 2370-2376.

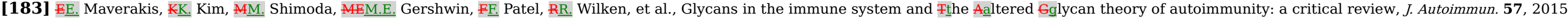
$1-13$.

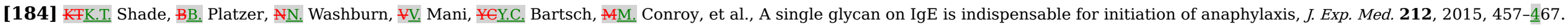

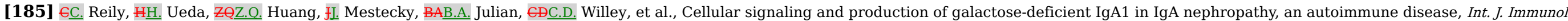
Res. 2014, 2014, 197548

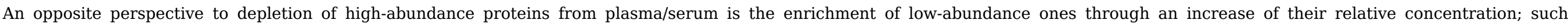

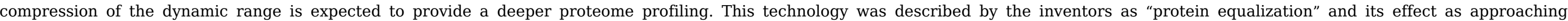

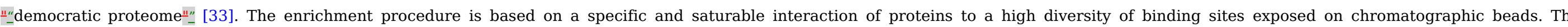

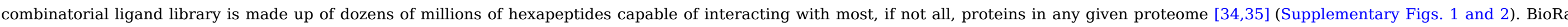

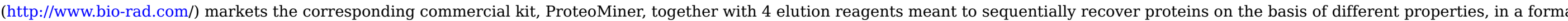

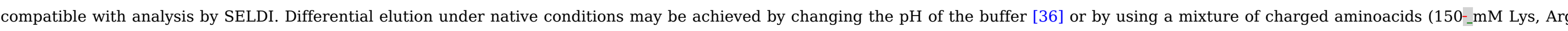

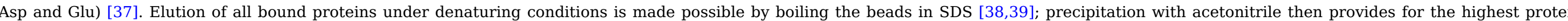
recovery yield and the best 2DE spot pattern [40].

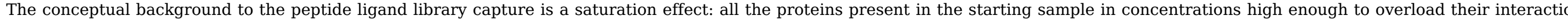

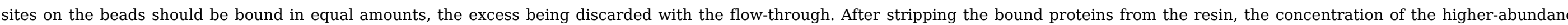

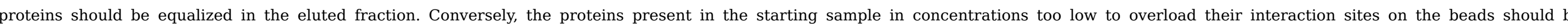

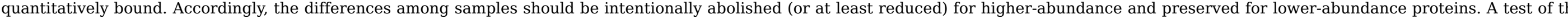

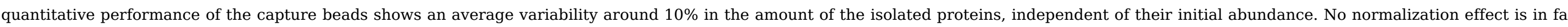




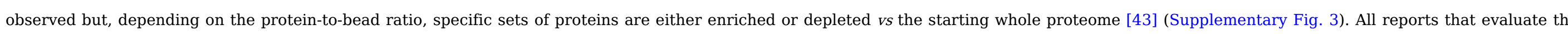

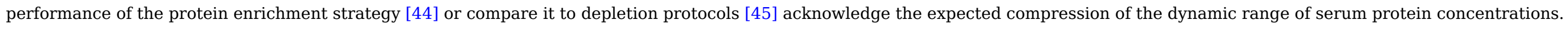

The following are the supplementary data related to this article.

\section{stepwise elution from combinatorial peptide ligand library beads}
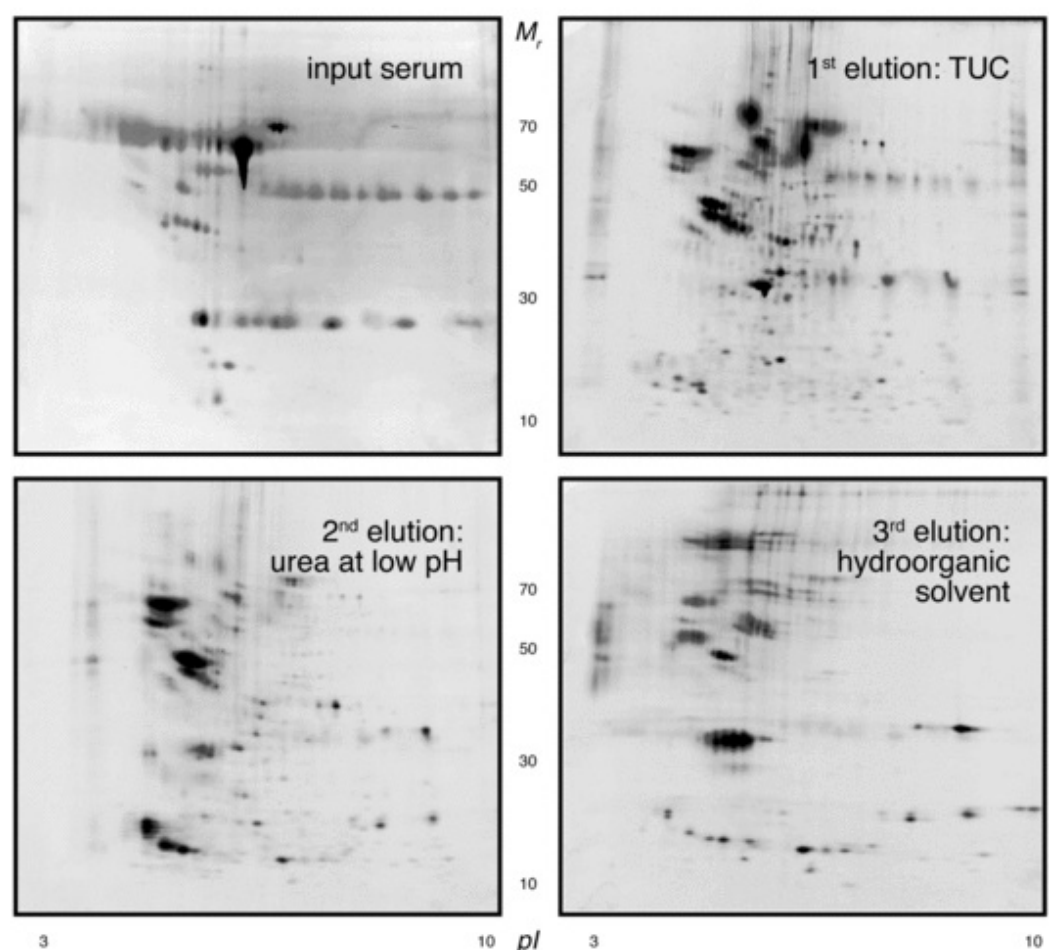

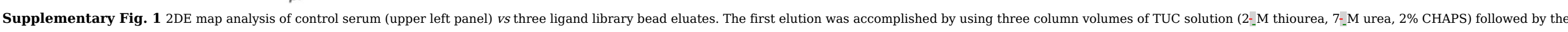

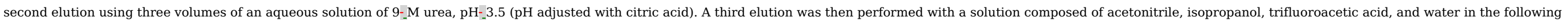
volumetric proportions: 16.6, 33.3, 0.5, and 49.5\%. (From [41]). 
size-dependence of binding by combinatorial peptide libraries

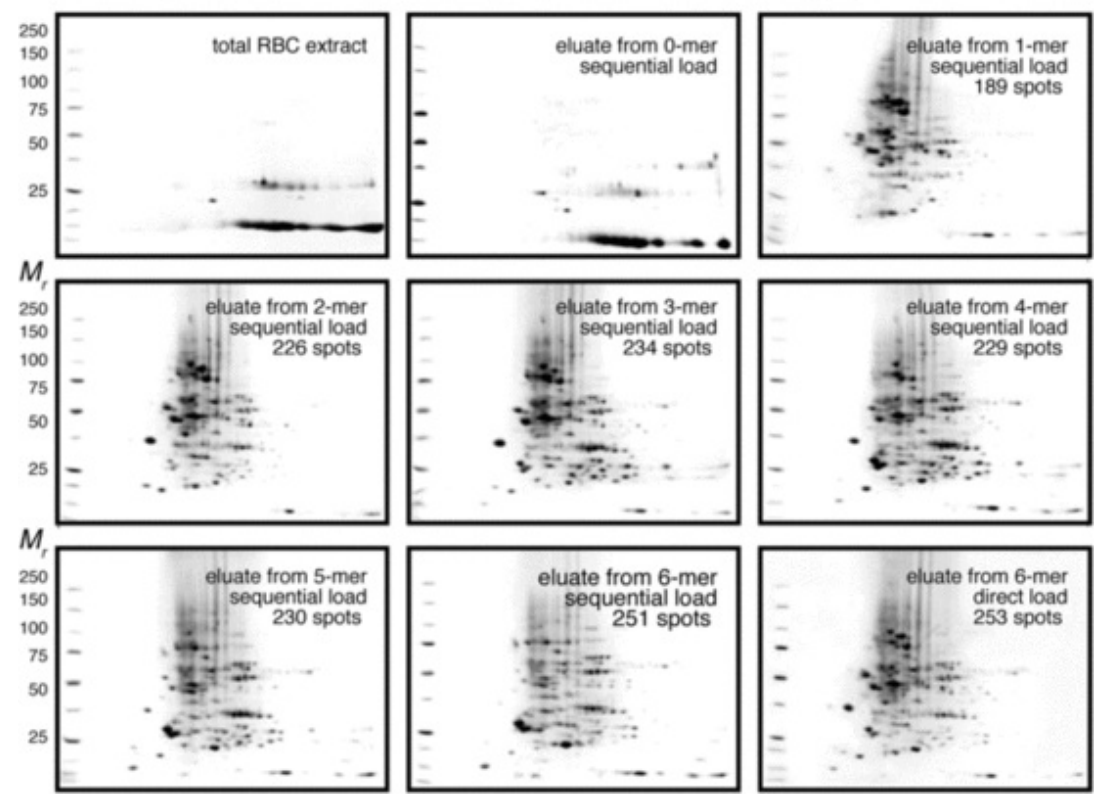

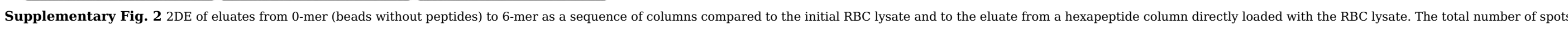
counted per map is marked. (From [42]).

alt-text: Supplementary Fig. 2

enrichment on combinatorial peptide ligand library beads
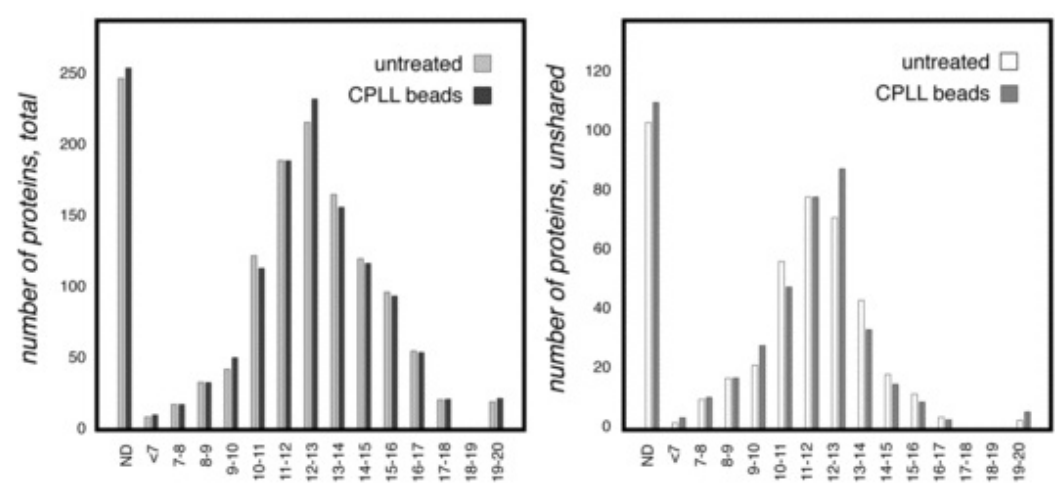

abundance ( $\log _{2}$ copies/cell)

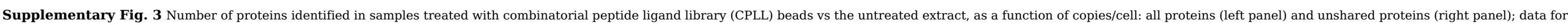
yeast cell proteins (S. cerevisiae strain S288C, BY4741). (Redrawn from [43].)

alt-text: Supplementary Fig. 3 


\section{Graphical abstract}

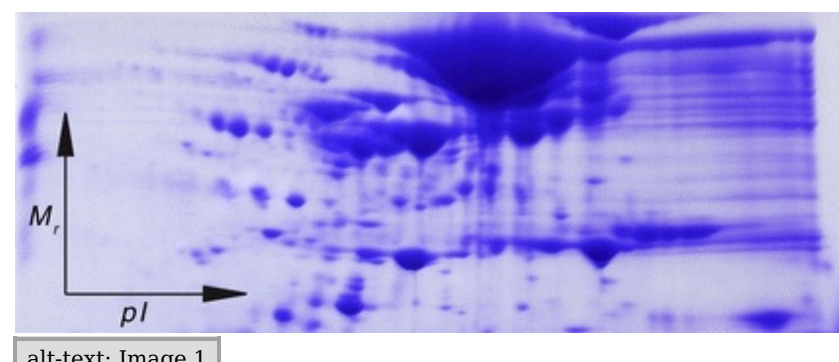

alt-text: Image 1

\section{Highlights}

- The review presents and discusses protocols for depletion of abundant serum proteins』

- It also presents approaches for the effective analysis of whole, untreated serum_

- Next it elaborates on multiple molecular forms-//multiple functions of serum proteins:

- It then recommends that information on abundant serum proteins be routinely obtained,

\section{Queries and Answers}

Query:

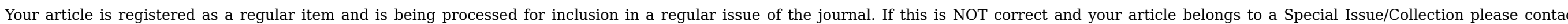
a.anwerbasha@elsevier.com immediately prior to returning your corrections.

Answer: Yes

Query:

The author names have been tagged as given names and surnames (surnames are highlighted in teal color). Please confirm if they have been identified correctly Answer: Yes 